\title{
THE GENERALIZED MARSHALL-OLKIN-KUMARASWAMY-G FAMILY OF DISTRIBUTIONS
}

\author{
Subrata Chakraborty ${ }^{1}$, Laba Handique ${ }^{2}$ \\ Department of Statistics, Dibrugarh University, Dibrugarh-786004, Assam, India \\ *Corresponding Author.Email: subrata_stats@dibru.ac.in
}

\begin{abstract}
A family of distribution is proposed by using Kumaraswamy-G ( $\mathrm{Kw}-\mathrm{G}$ ) distribution as the base line distribution in the generalized Marshall-Olkin (GMO) construction. By expanding the probability density function and the survival function as infinite series the proposed family is seen as infinite mixtures of the $\mathrm{Kw}-\mathrm{G}$ distribution. Series expansions of the density function for order statistics are also obtained. Moments, moment generating function, Rényi entropy, quantile function, random sample generation, asymptotes, shapes and stochastic orderings are also investigated. Maximum likelihood estimation, their large sample standard error, confidence intervals and method of moment are presented. Three real life illustrations of comparative data modeling applications with some of the important sub models of the family reveals the superiority of the proposed generalization over its sub models.
\end{abstract}

Key words: Marshall - Olkin -Kumaraswamy-G family, Generalized Marshall-Olkin family, Exponentiated family, Entropy, AIC and Power Weighted Moments.

\section{Introduction}

Generating new distributions starting with a base line distribution by adding one or more additional parameters through various mechanisms is an area of research in the filed of the probability distribution which have seen lot of work of late. The basic motivation of these works is to bring in more flexibility in the modelling different type of data generated from real life situation. Recently, there is renewed activity in this area to propose and investigate new families of distributions. A review paper by Tahir et al. (2015) provides a detail account of important techniques of generating new families of univariate continuous distributions through introduction of additional parameters. Some recent contributions since 2015 in this line include the Kumaraswamy Marshall-Olkin family proposed by Alizadeh et al. (2015), the Kumaraswamy Transmuted-G family of distributions (Afify et al., 2016), the Weibull generalized flexible Weibull extension distribution (Abdelfattah et al., 2016), the exponentiated generalized extended exponential distribution (Thiago et al., 2016), the generalized class of exponentiated modified Weibull distribution (Shusen et al., 2016), and Marshall-Olkin Kumaraswamy-G family introduced by Handique et al. (2017), the Modified Burr III G family of distributions (Shahzadi et al., 2017), Marshall-Olkin extended generalized Gompertz distribution (Lazhar, 2017) among others.

In this article we propose another family of continuous probability distribution as an extension of the Marshall-Olkin Kumaraswamy-G ( MOKw - G) family (Handique et al., 2017) and generalized Marshall-Olkin ( GMO ) family (Jayakumar and Mathew, 2008) by incorporating the Kumaraswamy-G ( $\mathrm{Kw}-\mathrm{G}$ ) family of distributions (Cordeiro and de Castro, 2011) as the base line distribution in the GMO family. This new family is referred to as the 
Generalized Marshall-Olkin Kumaraswamy-G ( GMOKw-G) family of distribution is investigated for some its general properties, parameter estimation and real life applications.

The rest of this article is organized in six sections. In section 2 we briefly introduce some important characteristics of probability distributions, the $\mathrm{GMO}$ and the $\mathrm{Kw}-\mathrm{G}$ family of distributions. The proposed new family is defined along with a genesis in section 3 . In section 4 we discuss some general results of the proposed family, while different methods of parameter estimation along with comparative data modelling examples are presented in section 5 . The article ends with a conclusion in section 6 followed by appendices of various proofs and derivations.

\section{Formulas and notations}

The different formulas to be used in the subsequent sections of this article are as follows:

If $T$ is a continuous random variable with pdf, $f(t)$ and cdf $F(t)=P[T \leq t]$, then

- Survival function (sf): $\bar{F}(t)=P[T>t]=1-F(t)$,

- Hazard rate function (hrf): $h(t)=f(t) / \bar{F}(t)$,

- Reverse hazard rate function (rhrf): $r(t)=f(t) / F(t)$,

- Cumulative hazard rate function (chrf): $H(t)=-\log [\bar{F}(t)]$,

- $(p, q, r)^{\mathrm{th}}$ Power Weighted Moment (PWM): $\Gamma_{\mathrm{p}, \mathrm{q}, \mathrm{r}}=\int_{-\infty}^{\infty} t^{\mathrm{p}}[F(t)]^{\mathrm{q}}[1-F(t)]^{\mathrm{r}} f(t) d t$,

- Rényi entropy: $I_{\mathrm{R}}(\delta)=(1-\delta)^{-1} \log \int_{-\infty}^{\infty} f(t)^{\delta} d t$.

\subsection{Generalized Marshall-Olkin Extended (GMO ) family of distribution}

Jayakumar and Mathew (2008) proposed a generalization of the Marshall-Olkin (MO) family of distributions (Marshall and Olkin, 1997) by using the Lehman second alternative (Lehmann 1953) to obtain the sf $\bar{F}^{\mathrm{GMO}}(t)$ of the GMO family of distributions by exponentiation the sf of MO family of distributions

$$
\bar{F}^{\mathrm{GMO}}(t)=[\{\alpha \bar{F}(t)\} /\{1-\bar{\alpha} \bar{F}(t)\}]^{\theta},-\infty<t<\infty, \theta>0, \alpha>0 ; \bar{\alpha}=1-\alpha
$$

where $\bar{F}(t)$ is the base line sf, $\theta$ and $\alpha$ are additional shape parameters. When $\theta=1$, $\bar{F}^{\mathrm{GMO}}(t)=\bar{F}^{\mathrm{MO}}(t)$ and for $\theta=\alpha=1, \bar{F}^{\mathrm{GMO}}(t)=\bar{F}(t)$. The cdf and pdf of the GMO distribution are respectively $F^{\mathrm{GMO}}(t)=1-[\{\alpha \bar{F}(t)\} /\{1-\bar{\alpha} \bar{F}(t)\}]^{\theta}$

and

$$
f^{\mathrm{GMO}}(t)=\left\{\theta \alpha^{\theta} f(t) \bar{F}(t)^{\theta-1}\right\} /[1-\bar{\alpha} \bar{F}(t)]^{\theta+1}
$$

Reliability measures like the hrf, rhrf and chrf associated with (1) are respectively given by

$$
\begin{aligned}
& h^{\mathrm{GMO}}(t)=\theta f(t) / \bar{F}(t)\{1-\bar{\alpha} \bar{F}(t)\}=\theta h(t) /\{1-\bar{\alpha} \bar{F}(t)\} \\
& r^{\mathrm{GMO}}(t)=\left\{\theta \alpha^{\theta} f(t) \bar{F}(t)^{\theta-1}\right\} /\left\{[1-\bar{\alpha} \bar{F}(t)]^{\theta+1}-\alpha^{\theta} \bar{F}(t)^{\theta}[1-\bar{\alpha} \bar{F}(t)]\right\}
\end{aligned}
$$


$H^{\mathrm{GMO}}(t)=-\log [\alpha \bar{F}(t) /\{1-\bar{\alpha} \bar{F}(t)\}]^{\theta}=-\theta \log [\alpha \bar{F}(t) /\{1-\bar{\alpha} \bar{F}(t)\}]$

where $f(t), F(t), \bar{F}(t)$ and $h(t)$ are respectively the pdf, cdf, sf and hrf of the baseline distribution. We denote the family of distribution having pdf in equation (2) as $\operatorname{GMO}(\theta, \alpha)$ which for $\theta=1$, reduces to $\mathrm{MO}(\alpha)$.

\subsection{Kumaraswamy-G (Kw - G) family of distribution}

The Kw $-\mathrm{G}(a, b)$ family of distribution of Cordeiro and de Castro (2011) for a baseline cdf $G(t)$ with pdf $g(t)$ is defined with respective cdf and pdf

$$
F^{\mathrm{KwG}}(t)=1-\left[1-G(t)^{a}\right]^{b}, \quad 0<t<\infty, 0<a, b<\infty
$$

and

$$
f^{\mathrm{KwG}}(t)=\operatorname{abg}(t) G(t)^{a-1}\left[1-G(t)^{a}\right]^{b-1} .
$$

where $t>0, g(t)=G^{\prime}(t)$ and $a>0, b>0$ are the shape parameters in addition to those in the baseline distribution. The sf, hrf, rhrf and chrf of this distribution are respectively given by

$$
\begin{aligned}
& \bar{F}^{\mathrm{KwG}}(t)=\left[1-G(t)^{a}\right]^{b}, h^{\mathrm{KwG}}(t)=\operatorname{abg}(t) G(t)^{a-1}\left[1-G(t)^{a}\right]^{-1} \\
& r^{\mathrm{KwG}}(t)=\operatorname{abg}(t) G(t)^{a-1}\left[1-G(t)^{a}\right]^{b-1}\left\{1-\left[1-G(t)^{a}\right]^{b}\right\}^{-1} \text { and } H^{\mathrm{KwG}}(t)=-b \log \left[1-G(t)^{a}\right] .
\end{aligned}
$$

\section{Generalized Marshall-Olkin Kumaraswamy-G (GMOKw - G) family of distribution}

We now propose a new extension of the GMO family by considering the cdf and pdf of $\mathrm{Kw}-\mathrm{G}$ distribution in (3) and (4) as the $f(t)$ and $F(t)$ respectively in the GMO formulation in (2) and call it GMOKw - G family of distribution and denote by GMOKw - G $(\theta, \alpha, a, b)$. The resulting expression for the pdf of $\mathrm{GMOKw}-\mathrm{G}(\theta, \alpha, a, b)$ is given by

$$
\begin{gathered}
f^{\mathrm{GMOKwG}}(t)=\left\{\theta \alpha^{\theta} \operatorname{abg}(t) G(t)^{a-1}\left[1-G(t)^{a}\right]^{b \theta-1}\right\} /\left[1-\bar{\alpha}\left[1-G(t)^{a}\right]^{b}\right]^{\theta+1} \\
, 0<t<\infty, \theta>0, \alpha>0,0<a, b<\infty .
\end{gathered}
$$

The cdf, sf, hrf, rhrf and chrf of GMOKw - G $(\theta, \alpha, a, b)$ distribution are respectively given by

$$
F^{\mathrm{GMOKwG}}(t)=1-\left[\frac{\alpha\left[1-G(t)^{a}\right]^{b}}{1-\bar{\alpha}\left[1-G(t)^{a}\right]^{b}}\right]^{\theta} \text { and } \bar{F}^{\mathrm{GMOKwG}}(t)=\left[\frac{\alpha\left[1-G(t)^{a}\right]^{b}}{1-\bar{\alpha}\left[1-G(t)^{a}\right]^{b}}\right]^{\theta}
$$

hrf:

$$
h^{\mathrm{GMOKwG}}(t)=\left\{\theta \operatorname{abg}(t) G(t)^{a-1}\left[1-G(t)^{a}\right]^{-1}\right\} /\left\{1-\bar{\alpha}\left[1-G(t)^{a}\right]^{b}\right\}
$$

rhrf: $\quad r^{\mathrm{GMOKwG}}(t)=\frac{\theta \alpha^{\theta} \operatorname{abg}(t) G(t)^{a-1}\left[1-G(t)^{a}\right]^{b \theta-1}}{\left[1-\bar{\alpha}\left[1-G(t)^{a}\right]^{b}\right]^{\theta+1}-\alpha^{\theta}\left[1-G(t)^{a}\right]^{b \theta}\left[1-\bar{\alpha}\left[1-G(t)^{a}\right]^{b}\right]}$

chrf: $\quad H^{\mathrm{GMOKwG}}(t)=-\theta \log \left[\left\{\alpha\left[1-G(t)^{a}\right]^{b}\right\} /\left\{1-\bar{\alpha}\left[1-G(t)^{a}\right]^{b}\right\}\right]$

We get for

(i) $\theta=1$, the MOKw-G $(\alpha, a, b)$ distribution of Handique et al. (2017) 
(ii) $\quad a=1$, a new family with cdf $1-\left[\frac{\alpha[\bar{G}(t)]^{b}}{1-\bar{\alpha}[\bar{G}(t)]^{b}}\right]^{\theta}$ is obtained. We refer to this family as the Generalized Marshall-Olkin exponentiated G-I in short as GMOexp - G - I $(\theta, \alpha, b)$ family.

(iii) $\quad b=1$, a new family with cdf $1-\left[\frac{\alpha\left[1-G(t)^{a}\right]}{1-\bar{\alpha}\left[1-G(t)^{a}\right]}\right]^{\theta}$ is obtained. We refer to this as the Generalized Marshall-Olkin exponentiated G-II in sort as GMOexp - G - I $(\theta, \alpha, a)$ family.

(iv) $a=b=1$, the $\operatorname{GMO}(\theta, \alpha)$ family of Jayakumar and Mathew (2008)

\subsection{Genesis of the distribution}

Here we provide a result to show how distributions from this proposed family may arise stochastically.

\section{Theorem1.}

For $i=1,2, \ldots, \theta$ where $\theta>1$ is an integer, if $\left\{T_{\mathrm{i} 1}, T_{\mathrm{i} 2}, \ldots, T_{\mathrm{iN}}\right\}$ be a sequence of i.i.d. random variables with survival function $\left[1-G(t)^{a}\right]^{b}$, and

i. if $N$ has a geometric distribution with parameter $\alpha(0<\alpha \leq 1)$ independent of $T_{i j}$ 's , then $\min _{1 \leq i \leq \theta}\left\{\min \left(T_{\mathrm{i} 1}, T_{\mathrm{i} 2}, \ldots, T_{\mathrm{iN}}\right)\right\}$ is distributed as GMOKw $-\mathrm{G}(\theta, \alpha, a, b)$ or

ii. if $N$ has a geometric distribution with parameter $1 / \alpha(\alpha>1)$ independent of $T_{i j}$ 's , then $\min _{1 \leq i \leq \theta}\left\{\max \left(T_{\mathrm{i} 1}, T_{\mathrm{i} 2}, \ldots, T_{\mathrm{iN}}\right)\right\}$ is distributed as $\mathrm{GMOKw}-\mathrm{G}(\theta, \alpha, a, b)$. (For proof see appendix A.1)

\subsection{Shape of the density and hazard function}

In this section we have plotted the pdf (in fig.1) and hrf (in fig. 2) of some members of GMOKw -E namely (a) GMOKw - $\mathrm{E}(\theta, \alpha, a, b, \lambda) \quad$ (b) $\mathrm{GMOKw}-\mathrm{W}(\theta, \alpha, a, b, \lambda, \delta)$ GMOKw $-\mathrm{L}(\theta, \alpha, a, b, \beta, \delta)$ and (d) GMOKw $-\operatorname{Fr}(\theta, \alpha, a, b, \lambda, \delta)$ by taking Exponential $(\lambda)$, Weibull $(\lambda, \delta)$, Lomax $(\beta, \delta)$ and Frechet $(\lambda, \delta)$ as the base line $\mathrm{G}$ in the GMOKw-G for some choices of the parameters to study the variety of shapes assumed by the family.

From the plots in figure 1 and 2 it can be seen that the family is very flexible and can offer many different types of shapes. It offers IFR, DFR even bath tub shaped hazard rate. 


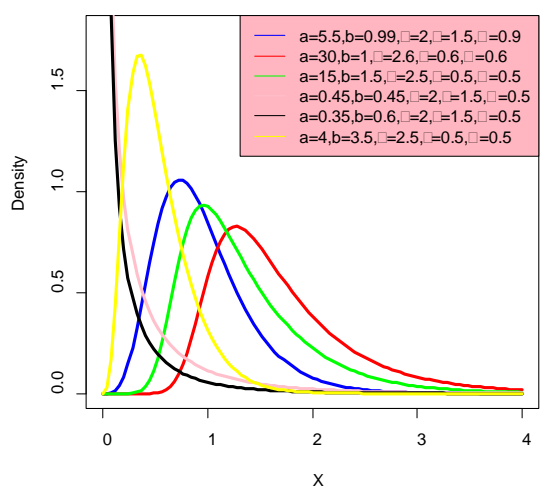

(a)

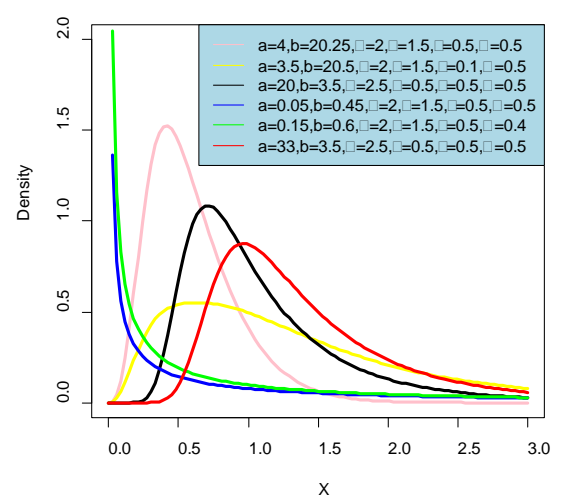

(c)

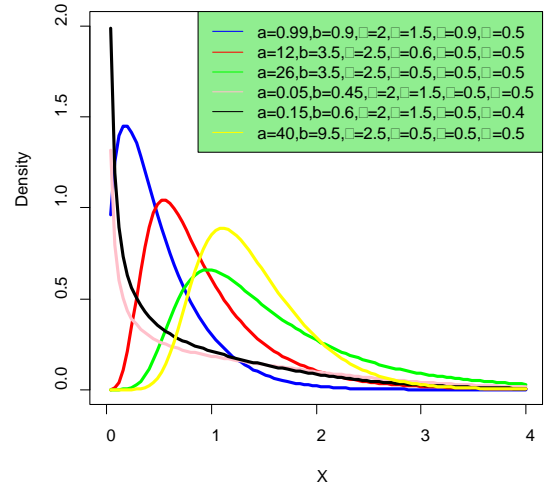

(b)

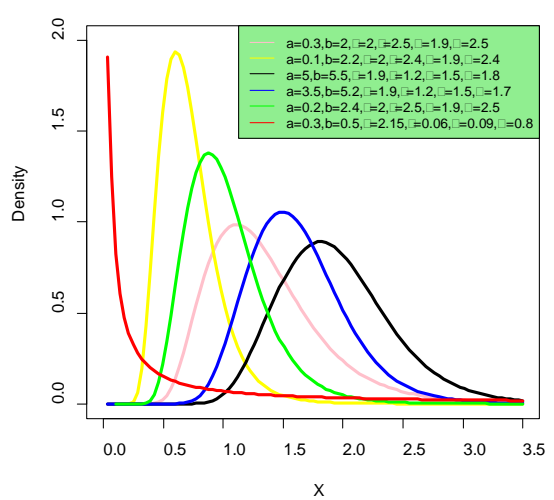

(d)

Fig 1: Density plots

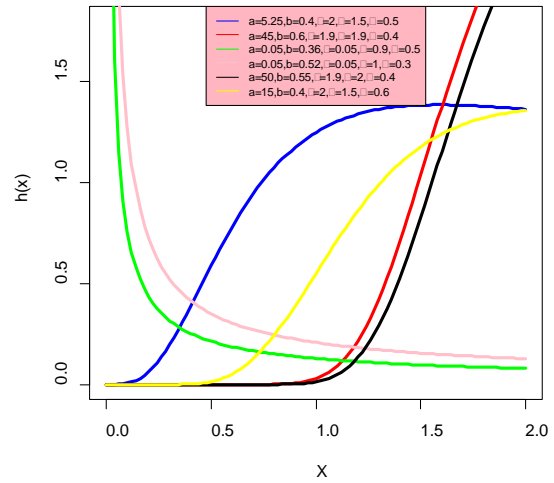

(a)

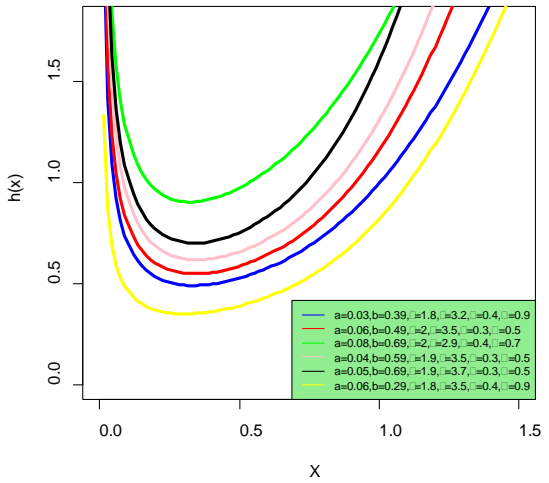

(b) 


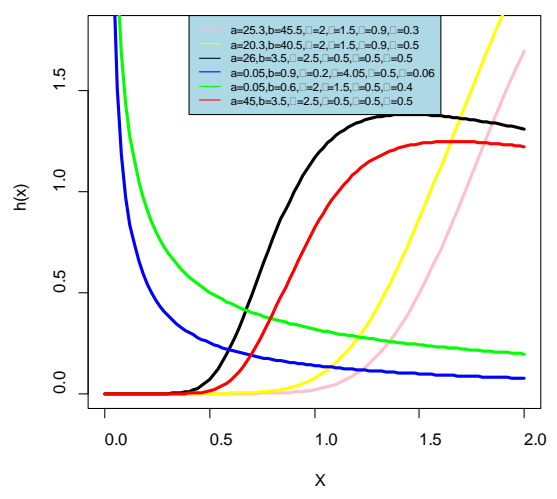

(c)

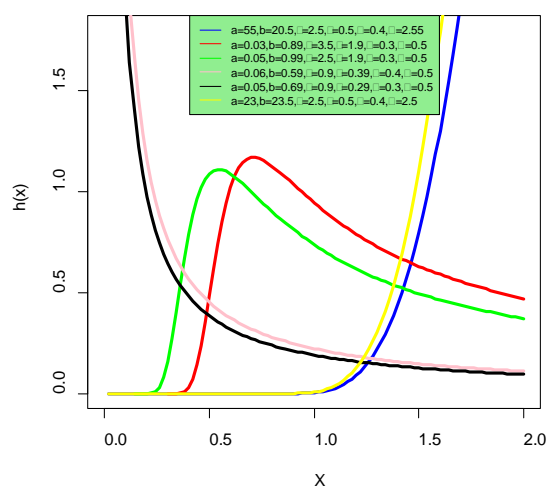

(d)

Fig 2: Hazard plots

\section{General results for GMOKw - G family}

Here some general results for the proposed GMOKw $-\mathrm{G}(\theta, \alpha, a, b)$ family are obtained in a similar manner as was done in Barreto-Souza et al. (2013), Cordeiro et al. (2014), and Alizadeh et al. (2015) among others.

\subsection{Expansions}

We know that $(1-z)^{-k}=\sum_{j=0}^{\infty} \frac{\Gamma(k+j)}{\Gamma(k) j !} z^{j},|z|<1$ and $k>0$

where $\Gamma($.$) is the gamma function.$

If $\alpha \in(0,1)$ using (8) in (5), we can show that

$$
\begin{aligned}
f^{\mathrm{GMOKwG}}(t) & =\theta \alpha^{\theta} \operatorname{abg}(t) G(t)^{a-1}\left[1-G(t)^{a}\right]^{b \theta-1}\left[1-\bar{\alpha}\left[1-G(t)^{a}\right]^{b}\right]^{-(\theta+1)} \\
& =f^{\mathrm{KwG}}(t ; a, b) \sum_{j=0}^{\infty} A_{j}\left[\bar{F}^{\mathrm{KwG}}(t ; a, b)\right]^{j+\theta-1} \\
& =-\sum_{j=0}^{\infty} A_{j}^{\prime} \frac{d}{d t}\left[\bar{F}^{\mathrm{KwG}}(t ; a, b)\right]^{j+\theta} \\
& =\sum_{j=0}^{\infty} A_{j}^{\prime} f^{\mathrm{KwG}}(t ; a, b(j+\theta))
\end{aligned}
$$

where $A_{j}^{\prime}=A_{j}^{\prime}(\alpha)=\left(\begin{array}{c}j+\theta-1 \\ j\end{array}\right)(1-\alpha)^{j} \alpha^{\theta}, \quad A_{j}=A_{j}(\alpha)=(j+\theta) A_{j}^{\prime}$

From this formulation the $\mathrm{GMOKw}-\mathrm{G}(\theta, \alpha, a, b)$ can be called a non central $\mathrm{Kw}-\mathrm{G}$ distribution. Similarly an expansion for the survival function of GMOKw-G $(\theta, \alpha, a, b)$ [for $\alpha \in(0,1)$ ] can be derived as 


$$
\bar{F}^{\mathrm{GMOKwG}}(t)=\sum_{j=0}^{\infty} A_{j}^{\prime} \bar{F}^{\mathrm{KwG}}(t ; a, b(j+\theta))
$$

Alternatively, we can also expand the pdf as

$$
f^{\mathrm{GMOKwG}}(t)=f^{\mathrm{KwG}}(t ; a, b \theta) \sum_{j=0}^{\infty} \sum_{k=0}^{j} B_{j, k}\left[F^{\mathrm{KwG}}(t ; a, b)\right]^{j-k}
$$

where $B_{j, k}=B_{j, k}(\alpha)=\frac{\theta \alpha^{\theta}(j+\theta) !}{\theta ! j !}(1-\alpha)^{j}(-1)^{j-k}\left(\begin{array}{l}j \\ k\end{array}\right)=(-1)^{j-k}\left(\begin{array}{l}j \\ k\end{array}\right) A_{j}$

Another expansion of the density function in (5) can be obtained for $\alpha>1$ using (8) as $f^{\mathrm{GMOKwG}}(t)=f^{\mathrm{KwG}}(t ; a, b \theta) \sum_{j=0}^{\infty} C_{j}\left\{F^{\mathrm{KwG}}(t ; a, b)\right\}^{j}$

where $C_{j}=C_{j}(\alpha)=\frac{\theta(j+\theta) !}{\theta ! j ! \alpha}\left(1-\frac{1}{\alpha}\right)^{j}=\frac{(-1)^{j-1}}{\alpha^{\theta+j+1}} A_{j}$

Corresponding expansion for the survival function of GMOKw - G $(\theta, \alpha, a, b)$ can be written as $\bar{F}^{\mathrm{GMOKwG}}(t)=\left[\bar{F}^{\mathrm{KwG}}(t ; a, b \theta)\right] \sum_{j=0}^{\infty} C_{j}^{\prime}\left[F^{\mathrm{KwG}}(t ; a, b)\right]^{j}$, where $C_{j}^{\prime}=(\alpha / j+\theta) C_{j}$.

\subsection{Order statistics}

Given a random sample $T_{1}, T_{2}, \ldots, T_{n}$ from any GMOKw - G $(\theta, \alpha, a, b)$ distribution the pdf of $T_{i: n}$ the $i^{\text {th }}$ order statistics can be derived as (See Appendix A.2 for the derivation)

$$
\begin{aligned}
f_{i: n}(t) & =\{n ! /(i-1) !(n-i) !\} f^{\mathrm{GMOKwG}}(t)\left[1-\bar{F}^{\mathrm{GMOKwG}}(t)\right]^{i-1} \bar{F}^{\mathrm{GMOKwG}}(t)^{n-i} \\
& =\{n ! /(i-1) !(n-i) !\} f^{\mathrm{GMOKwG}}(t) \sum_{l=0}^{i-1}(-1)^{l}\left(\begin{array}{c}
i-1 \\
l
\end{array}\right) \bar{F}^{\mathrm{GMOKwG}}(t)^{n+l-i}
\end{aligned}
$$

Now on using the general expansions of the pdf and sf of the GMOKw - G $(\theta, \alpha, a, b)$ distribution, we get the corresponding pdf of the $i^{\text {th }}$ order statistics for of the GMOKw $-\mathrm{G}(\theta, \alpha, a, b)$ for $\alpha \in(0,1)$ as

$$
\begin{aligned}
f_{i: n}(t) & =f^{\mathrm{KwG}}(t ; a, b) \sum_{j, p=0}^{\infty} M_{j, p}\left[\bar{F}^{\mathrm{KwG}}(t ; a, b)\right]^{j+p+\theta(n+l-i+1)-1} \\
& \left.=\sum_{j, p=0}^{\infty} M_{j, p}^{\prime} f^{\mathrm{KwG}}(t ; a, b(j+p+\theta(n+l-i+1)))\right]
\end{aligned}
$$

where $M_{j, p}=n A_{j} A_{p}^{\prime}\left(\begin{array}{c}n-1 \\ i-1\end{array}\right) \sum_{l=0}^{i-1}\left(\begin{array}{c}i-1 \\ l\end{array}\right)(-1)^{l+1}, M_{j, p}^{\prime}=M_{j, p} /(j+p+\theta(n+l-i+1))$ and $A_{j}$ defined and $A_{j}^{\prime}$ are earlier. 
In particular for $\theta=1$, equation (13) reduces to pdf of the $i^{\text {th }}$ order statistics for of the MOKw - G $(\alpha, a, b)$ (Handique et al., 2017) given by

$$
\begin{gathered}
f_{i: n}(t) \\
H_{j, c}=n \kappa_{j} \eta_{c}\left(\begin{array}{c}
n-1 \\
i-1
\end{array}\right) \sum_{l=0}^{i-1}\left(\begin{array}{c}
i-1 \\
l
\end{array}\right)(-1)^{l+1} \text { and } \kappa_{j}=\kappa_{j}(\alpha)=(j+1) \alpha(1-\alpha)^{j} ; \eta_{c}=\alpha(1-\alpha)^{c} H_{j, c}\left[\bar{F}^{\mathrm{KwG}}(t ; a, b)\right]^{j+c+n+l-i}
\end{gathered}
$$

Further using the general expansion of the pdf and sf of the GMOKw - G $(\theta, \alpha, a, b)$ distribution we get the pdf of the $i^{\text {th }}$ order statistics for of the GMOKw $-\mathrm{G}(\theta, \alpha, a, b)$ for $\alpha>1$ as

$$
\begin{aligned}
& f_{i: n}(t)=f^{\mathrm{KwG}}(t ; a, b)\left[\bar{F}^{\mathrm{KwG}}(t ; a, b)\right]^{\theta(n+l-i+1)-1} \sum_{j, k=0}^{\infty} \phi_{j, k}\left\{F^{\mathrm{KwG}}(t ; a, b)\right\}^{j+k} \\
& \text { where } \phi_{j, k}=n C_{j} d_{n+l-i, k}\left(\begin{array}{c}
n-1 \\
i-1
\end{array}\right) \sum_{l=0}^{i-1}(-1)^{l}\left(\begin{array}{c}
i-1 \\
l
\end{array}\right), \\
& \qquad d_{n+l-i, k}=\frac{1}{k C_{0}^{\prime}} \sum_{h=1}^{k}[h(n+l-i-1)-k] C_{h}^{\prime} d_{n+l-i, k-h} \text { and } C_{j}, C_{k}^{\prime} \text { defined in section 4.1. }
\end{aligned}
$$

\section{Remark1}

From the equations in (9) to (14) we see that the density functions of the GMOKw $-\mathrm{G}(\theta, \alpha, a, b)$ distribution and that of its order statistics can be expressed as different $\mathrm{Kw}-\mathrm{G}$ density multiplied by an infinite power series of cdf of and also as negative binomial mixture of $\mathrm{Kw}-\mathrm{G}$ distributions under Lehman alternatives. Thus explicit expressions for the moments and moment generating function (mgf) of the GMOKw $-\mathrm{G}(\theta, \alpha, a, b)$ distribution and those of its order statistics in a general set up and for special models may be obtained using the corresponding results of $\mathrm{Kw}-\mathrm{G}$ distributions.

\subsection{Moments of GMOKw - G}

Greenwood et al. (1979) first proposed the probability weighted moments (PWMs), as expectations of certain functions of a random variable whose mean exists. They defined the $(p, q, r)^{\text {th }}$ PWM of $T$ as

$$
\Gamma_{p, q, r}=\int_{-\infty}^{\infty} t^{p}[F(t)]^{q}[1-F(t)]^{r} f(t) d t
$$

The PWM are useful in estimating parameters quantiles of generalized distributions and have low variance and moderate biases, and comparable with the maximum likelihood estimators.

The $s^{\text {th }}$ moment of $T$ for $\alpha \in(0,1)$ and $\alpha>1$, can be obtained from equations (9), (11) and (12), either as 
$\mathrm{E}\left(T^{s}\right)=\int_{-\infty}^{+\infty} t^{s} \operatorname{abg}(t) G(t)^{a-1}\left[1-G(t)^{a}\right]^{b-1} \sum_{j=0}^{\infty} A_{j}\left[\left\{1-G(t)^{a}\right\}^{b}\right]^{j+\theta-1} d t=\sum_{j=0}^{\infty} A_{j} \Gamma_{s, 0, j+\theta-1}$ or as $\mathrm{E}\left(T^{s}\right)=\int_{-\infty}^{+\infty} t^{s} \operatorname{abg}(t) G(t)^{a-1}\left[1-G(t)^{a}\right]^{b \theta-1} \sum_{j=0}^{\infty} C_{j}\left[1-\left[1-G(t)^{a}\right]^{b}\right]^{j} d t=\sum_{j=0}^{\infty} C_{j} \Gamma_{s, j, \theta-1}$

where $\Gamma_{p, q, r}=\int_{-\infty}^{\infty} t^{p}\left\{1-\left[1-G(t)^{a}\right]^{b}\right\}^{q}\left\{\left[1-G(t)^{a}\right]^{b}\right\}^{r}\left[\operatorname{abg}(t) G(t)^{a-1}\left[1-G(t)^{a}\right]^{b-1}\right] d t$ is the PWM of the $\mathrm{Kw}-\mathrm{G}(a, b)$ distribution.

Therefore the moments of the GMOKw $-\mathrm{G}(\theta, \alpha, a, b)$ can be expressed in terms of the PWMs of Kw-G $(a, b)$.

Similarly proceeding we can derive $s^{\text {th }}$ moment of the $i^{\text {th }}$ order statistic $T_{i: n}$, in a random sample of size $n$ from GMOKw - G $(\theta, \alpha, a, b)$ for $\alpha \in(0,1)$ and $\alpha>1$, by using equations (13) and (14) as

$$
E\left(T_{i, n}^{s}\right)=\sum_{j, p=0}^{\infty} M_{j, p} \Gamma_{s, 0, j+p+\theta(n+l-i+1)-1} \text { and } E\left(T_{i, n}^{s}\right)=\sum_{j, k=0}^{\infty} \phi_{j, k} \Gamma_{s, j+k, \theta(n+l-i+1)-1}
$$

where $A_{j}, B_{j, k}, C_{j}$ and $M_{j, p}, \phi_{j, k}$ defined in section 4.1 and 4.2 respectively.

\subsection{Moment generating function}

Here the mgf of GMOKw $-\mathrm{G}(\theta, \alpha, a, b)$ family is expressed in terms of that of the exponentiated $\mathrm{Kw}-\mathrm{G}$ distribution utilizing the results of section 4.1. For example using equation (10) we can show that

$$
\begin{aligned}
& M_{T}(s)=E\left[e^{s T}\right]=\int_{-\infty}^{\infty} e^{s t} f(t) d t=-\int_{-\infty}^{\infty} e^{s t} \sum_{j=0}^{\infty} A_{j}^{\prime} \frac{d}{d t}\left[\bar{F}^{K w G}(t ; a, b)\right]^{j+\theta} d t \\
& =-\sum_{j=0}^{\infty} A_{j}^{\prime} \int_{-\infty}^{\infty} e^{s t} \frac{d}{d t}\left\{\bar{F}^{K w G}(t ; a, b)\right\}^{j+\theta} d t=\sum_{j=0}^{\infty} A_{j} M_{X}(s)
\end{aligned}
$$

where $M_{X}(s)$ is the mgf of a $\mathrm{Kw}-\mathrm{G}$ with parameters $a$ and $b(j+\theta)$ distribution.

\subsection{Entropy}

In this subsection we present results on Rényi entropy and relative entropy.

\subsubsection{Rényi entropy}

The entropy of a random variable is a measure of uncertainty variation and has been used in different situations in science and engineering. Here, we give the Rényi entropy for the GMOKw - G $(\theta, \alpha, a, b)$ distribution. The Rényi entropy of a random variable with pdf $f(t)$ is defined as 


$$
I_{R}(\delta)=(1-\delta)^{-1} \log \left(\int_{-\infty}^{\infty} f(t)^{\delta} d t\right)
$$

where $\delta>0$ and $\delta \neq 1$. For furthers details, see Song (2001).

When $\alpha \in(0,1)$ using expansion (8), in (5) we can write

$$
\begin{aligned}
& f^{\mathrm{GMOKwG}}(t)^{\delta}=\left[\left\{\theta \alpha^{\theta} \operatorname{abg}(t) G(t)^{a-1}\left[1-G(t)^{a}\right]^{b \theta-1}\right\} /\left[1-\bar{\alpha}\left[1-G(t)^{a}\right]^{b}\right]^{\theta+1}\right]^{\delta} \\
& =\left[\theta \alpha^{\theta} \operatorname{abg}(t) G(t)^{a-1}\left\{1-G(t)^{a}\right\}^{b \theta-1}\right]^{\delta}\left[1-\bar{\alpha}\left[1-G(t)^{a}\right]^{b}\right]^{-\delta(\theta+1)} \\
& =\frac{\theta^{\delta} \alpha^{\delta \theta}\left[\operatorname{abg}(t) G(t)^{a-1}\left\{1-G(t)^{a}\right\}^{b \theta-1}\right]^{\delta}}{\Gamma[\delta(\theta+1)]} \sum_{j=0}^{\infty}(1-\alpha)^{j} \Gamma[\delta(\theta+1)+j] \frac{\left[\left\{1-G(t)^{a}\right\}^{b}\right]^{j}}{j !}
\end{aligned}
$$

Thus for $\alpha \in(0,1)$, the Rényi entropy of GMOKw - G $(\theta, \alpha, a, b)$ can be obtained as

$$
I_{R}(\delta)=(1-\delta)^{-1} \log \left(\sum_{j=0}^{\infty} L_{j} \int_{-\infty}^{\infty}\left[a b g(t) G(t)^{a-1}\left\{1-G(t)^{a}\right\}^{b \theta-1}\right]^{\delta}\left[\left\{1-G(t)^{a}\right\}^{b}\right]^{j} d t\right)
$$

where $L_{j}=L_{j}(\alpha)=\left\{\theta^{\delta} \alpha^{\delta \theta}(1-\alpha)^{j} \Gamma[\delta(\theta+1)+j]\right\} /\{\Gamma[\delta(\theta+1)] j !\}$

Again the density function (5) can be expressed as

$$
f^{\mathrm{GMOKwG}}(t)^{\delta}=\left[\left\{\theta a b g(t) G(t)^{a-1}\left[1-G(t)^{a}\right]^{b \theta-1} / \alpha\right\}\left[1-\left\{(\alpha-1)\left[1-\left[1-G(t)^{a}\right]^{b}\right]\right\} / \alpha\right]^{\theta+1}\right]^{\delta}
$$

For $\alpha>1$, using expansion (8) we get,

$$
\begin{aligned}
& =\left\{\left\{\theta \operatorname{abg}(t) G(t)^{a-1}\left[1-G(t)^{a}\right]^{b \theta-1} / \alpha\right\}\left[1-\left\{(\alpha-1)\left[1-\left[1-G(t)^{a}\right]^{b}\right]\right\} / \alpha\right]^{-(\theta+1)}\right\}^{\delta} \\
& =\frac{\left[\theta \operatorname{abg}(t) G(t)^{a-1}\left[1-G(t)^{a}\right]^{b \theta-1}\right]^{\delta}}{\alpha^{\delta+j} \Gamma[\delta(\theta+1)]} \sum_{j=0}^{\infty} \Gamma[\delta(\theta+1)+j](\alpha-1)^{j} \frac{\left[1-\left[1-G(t)^{a}\right]^{b}\right]^{j}}{j !}
\end{aligned}
$$

Thus for $\alpha>1$, the Rényi entropy of GMOKw $-\mathrm{G}(\theta, \alpha, a, b)$ also can be derived as

$$
I_{R}(\delta)=(1-\delta)^{-1} \log \left(\sum_{j=0}^{\infty} R_{j} \int_{-\infty}^{\infty}\left[\operatorname{abg}(t) G(t)^{a-1}\left[1-G(t)^{a}\right]^{b \theta-1}\right]^{\delta}\left[1-\left[1-G(t)^{a}\right]^{b}\right]^{j} d t\right)
$$

where $R_{j}=R_{j}(\alpha)=\left\{\theta^{\delta}(\alpha-1)^{j} \Gamma[\delta(\theta+1)+j]\right\} /\left\{\alpha^{\delta+j} \Gamma[\delta(\theta+1)] j !\right\}$.

\subsubsection{Relative entropy}

Given two distributions with pdfs $f_{1}(t)$ and $f_{2}(t)$ the relative entropy (RE) between them is defined as

$$
\operatorname{RE}\left(f_{1}, f_{2}\right)=E_{f_{1}}\left(\log \left\{f_{1}(t) / f_{2}(t)\right\}\right)=\int\left(\log \left\{f_{1}(t) / f_{2}(t)\right\}\right) f_{1}(t) d t .
$$

This is also referred to as Kullback-Leibler divergence (distance) (Kullback, 1959). $\mathrm{RE}$ is a measure of distance between $f_{1}(t)$ and $f_{2}(t)$ and measures inefficiency of selecting $f_{2}$ for modeling when $f_{1}$ is the true distribution. We have estimated $\operatorname{RE}\left(G M O K w-G, f_{2}\right)$ where $f_{2}$ is a competing distribution for all the data fitting examples considered in section 5 in Tables 1, 2, 3, 4, 5 and 6. 


\subsection{Quantile function, median and random sample generation}

For GMOKw-G $(\theta, \alpha, a, b)$ the $p^{\text {th }}$ Quantile $t_{p}$ is derived by solving the equation $F^{\mathrm{GMOKwG}}\left(t_{p}\right)=p$ as

$$
t_{p}=G^{-1}\left[1-\left\{[1-p]^{1 / \theta} /\left\{\alpha+\bar{\alpha}[1-p]^{1 / \theta}\right\}\right\}^{1 / b}\right]^{1 / a}
$$

Now a random number ' $t$ ' from GMOKw $-\mathrm{G}(\theta, \alpha, a, b)$ can be easily generated starting with a uniform random number ' $u$ ' using the formula

$t=G^{-1}\left[1-\left\{[1-u]^{1 / \theta} /\left\{\alpha+\bar{\alpha}[1-u]^{1 / \theta}\right\}\right\}^{1 / b}\right]^{1 / a}$

Median: $\quad t=G^{-1}\left[1-\left\{[1-0.5]^{1 / \theta} /\left\{\alpha+\bar{\alpha}[1-0.5]^{1 / \theta}\right\}\right\}^{1 / b}\right]^{1 / a}$

e.g. considering the exponential with parameter $\lambda>0$, as the base line distribution having pdf and cdf as $g(t: \lambda)=\lambda \exp (-\lambda t), t>0$ and $G(t: \lambda)=1-\exp (-\lambda t)$, respectively, the $p^{\text {th }}$ quantile $t_{p}$ of $G M O K w-E(\theta, \alpha, a, b, \lambda)$ is seen as

$t_{p}=-(1 / \lambda) \log \left[1-\left[1-\left\{[1-p]^{1 / \theta} /\left\{\alpha+\bar{\alpha}[1-p]^{1 / \theta}\right\}\right\}^{1 / b}\right]^{1 / a}\right]$.

\subsection{Asymptotes and shapes}

In this section we study the asymptotic shapes of the proposed family following the methods followed in Alizadeh et al., (2015).

\section{Proposition 2.}

The asymptotes of pdf, cdf and hrf of GMOKw - G $(\theta, \alpha, a, b)$ in equations (5), (6) and (7) as $t \rightarrow 0$ are given by

$$
\begin{aligned}
f(t) & \sim \operatorname{\theta abg}(t) G(t)^{a-1} / \alpha & & \text { as } t \rightarrow 0 \\
F(t) & \sim 0 & \text { as } t & \rightarrow 0 \\
h(t) & \sim \theta \operatorname{\theta abg}(t) G(t)^{a-1} / \alpha & \text { as } t & \rightarrow 0
\end{aligned}
$$

\section{Proposition 3.}

The asymptotes of pdf, cdf and hrf of GMOKw - G $(\theta, \alpha, a, b)$ in equations (5), (6) and (7) as $t \rightarrow \infty$ are given by

$$
\begin{array}{ll}
f(t) \sim \theta \alpha^{\theta} \operatorname{abg}(t)\left[1-G(t)^{a}\right]^{b \theta-1} & \text { as } t \rightarrow \infty \\
F(t) \sim 1-\alpha^{\theta}\left[1-G(t)^{a}\right]^{b \theta} & \text { as } t \rightarrow \infty \\
h(t) \sim \theta a b g(t)\left[1-G(t)^{a}\right]^{-1} & \text { as } t \rightarrow \infty
\end{array}
$$

The shapes of the pdf and hrf can be studied analytically. The critical points of the pdf of GMOKw - $\mathrm{G}(\theta, \alpha, a, b)$ are the roots of the equation.

$d \log [f(t)] / d t=g^{\prime}(t) / g(t)+\{(a-1) g(t)\} / G(t)+\left\{a(1-b \theta) g(t) G(t)^{a-1}\right\} /\left\{1-G(t)^{a}\right\}$ 
$-\left\{(\theta+1) \bar{\alpha} \operatorname{abg}(t) G(t)^{a-1}\left[1-G(t)^{a}\right]^{b-1}\right\} /\left\{1-\bar{\alpha}\left[1-G(t)^{a}\right]^{b}\right\}=0$

The equation (15) may have more than one roots. If $t=t_{0}$ is a root of (15) then it corresponds to either a local maximum, or a local minimum or a point of inflexion depending on whether $\lambda\left(t_{0}\right)<0, \lambda\left(t_{0}\right)>0$ or $\lambda\left(t_{0}\right)=0$ where $\lambda(t)=d^{2} / d t^{2} \log [f(t)]$. Here

$$
\begin{aligned}
& \lambda(t)=\left\{g(t) g^{\prime \prime}(t)-\left[g^{\prime}(t)\right]^{2}\right\} / g(t)^{2}+\left\{(a-1) G(t) g^{\prime}(t)-g(t)^{2}\right\} / G(t) \\
& +a(1-b \theta)\left\{1-G(t)^{a}\right\}^{-1}\left[g^{\prime}(t) G(t)^{a-1}+(a-1) g(t)^{2} G(t)^{a-2}+a g(t)^{2} G(t)^{2 a-2}\left\{1-G(t)^{a}\right\}^{-1}\right] \\
& -\frac{(\theta+1) \bar{\alpha} a b\left[g^{\prime}(t) G(t)^{a-1}\left[1-G(t)^{a}\right]^{b-1}\right]}{1-\bar{\alpha}\left[1-G(t)^{a}\right]^{b}}-\frac{(\theta+1) \bar{\alpha} a b\left[(a-1) g(t)^{2} G(t)^{a-2}\left[1-G(t)^{a}\right]^{b-1}\right]}{1-\bar{\alpha}\left[1-G(t)^{a}\right]^{b}} \\
& +\frac{(\theta+1) \bar{\alpha} a b\left[a(b-1) g(t)^{2} G(t)^{2 a-2}\left[1-G(t)^{a}\right]^{b-2}\right]}{1-\bar{\alpha}\left[1-G(t)^{a}\right]^{b}}+(\theta+1)\left[\frac{\bar{\alpha} a b g(t) G(t)^{a-1}\left[1-G(t)^{a}\right]^{b-1}}{1-\bar{\alpha}\left[1-G(t)^{a}\right]^{b}}\right]^{2} \\
& =\left\{g(t) g^{\prime \prime}(t)-\left[g^{\prime}(t)\right]^{2}\right\} / g(t)^{2}+\left\{(a-1) G(t) g^{\prime}(t)-g(t)^{2}\right\} / G(t) \\
& +a(1-b \theta)\left[\frac{g^{\prime}(t) G(t)^{a-1}}{1-G(t)^{a}}+\frac{(a-1) g(t)^{2} G(t)^{a-2}}{1-G(t)^{a}}+\frac{a g(t)^{2} G(t)^{2 a-2}}{\left.\left[1-G(t)^{a}\right]^{2}\right]}\right. \\
& -\frac{(\theta+1) \bar{\alpha} g^{\prime}(t)}{\theta g(t)}\left[1-G(t)^{a}\right]^{b} h^{G M O K w G}(t) \\
& +\{(\theta+1) / \theta\} \bar{\alpha} a(b-1) g(t) G(t)^{a-1}(t)\left\{1-G(t)^{a}\right\}^{b-1} h^{G M O K w G}(t) \\
& +\left\{(\theta+1) / \theta^{2}\right\}\left\{\left[1-G(t)^{a}\right]^{b} \bar{\alpha} h^{G M O K w G}(t)\right\}^{2}
\end{aligned}
$$

The critical points of hrf are the roots of the equation

$$
\frac{d \log [h(t)]}{d t}=\frac{g^{\prime}(t)}{g(t)}+(a-1) \frac{g(t)}{G(t)}+\frac{a g(t) G(t)^{a-1}}{1-G(t)^{a}}-\frac{\bar{\alpha} a b g(t) G(t)^{a-1}\left[1-G(t)^{a}\right]^{b-1}}{1-\bar{\alpha}\left[1-G(t)^{a}\right]^{b}}=0
$$

Here also the equation (16) may have more than one root. If $t=t_{0}$ is a root of (16) then it corresponds to either a local maximum, or a local minimum or a point of inflexion depending on whether

$$
\begin{aligned}
& \gamma\left(t_{0}\right)<0, \gamma\left(t_{0}\right)>0 \text { or } \gamma\left(t_{0}\right)=0 \text {, where } \gamma(t)=d^{2} \log [h(t)] / d t^{2} \text {. Here } \\
& \begin{aligned}
& \gamma(t)=\left\{g(t) g^{\prime \prime}(t)-\left[g^{\prime}(t)\right]^{2}\right\} / g(t)^{2}+\left\{(a-1) G(t) g^{\prime}(t)-g(t)^{2}\right\} / G(t) \\
&+a\left[\frac{g^{\prime}(t) G(t)^{a-1}}{1-G(t)^{a}}+\frac{(a-1) g(t)^{2} G(t)^{a-2}}{1-G(t)^{a}}+\frac{a g(t)^{2} G(t)^{2 a-2}}{\left[1-G(t)^{a}\right]^{2}}\right] \\
&-\frac{\bar{\alpha} a b\left[g^{\prime}(t) G(t)^{a-1}\left[1-G(t)^{a}\right]^{b-1}\right]}{1-\bar{\alpha}\left[1-G(t)^{a}\right]^{b}}-\frac{\bar{\alpha} a b\left[(a-1) g(t)^{2} G(t)^{a-2}\left[1-G(t)^{a}\right]^{b-1}\right]}{1-\bar{\alpha}\left[1-G(t)^{a}\right]^{b}} \\
&+\frac{\bar{\alpha} a b\left[a(b-1) g(t)^{2} G(t)^{2 a-2}\left[1-G(t)^{a}\right]^{b-2}\right]}{1-\bar{\alpha}\left[1-G(t)^{a}\right]^{b}}+\left[\frac{\bar{\alpha} a b g(t) G(t)^{a-1}\left[1-G(t)^{a}\right]^{b-1}}{1-\bar{\alpha}\left[1-G(t)^{a}\right]^{b}}\right]^{2} \\
&=\left\{g(t) g^{\prime \prime}(t)-\left[g^{\prime}(t)\right]^{2}\right\} / g(t)^{2}+\left\{(a-1) G(t) g^{\prime}(t)-g(t)^{2}\right\} / G(t)
\end{aligned}
\end{aligned}
$$




$$
\begin{aligned}
& +a\left[\frac{g^{\prime}(t) G(t)^{a-1}}{1-G(t)^{a}}+\frac{(a-1) g(t)^{2} G(t)^{a-2}}{1-G(t)^{a}}+\frac{a g(t)^{2} G(t)^{2 a-2}}{\left[1-G(t)^{a}\right]^{2}}\right]-\frac{\bar{\alpha} g^{\prime}(t)}{\theta g(t)}\left[1-G(t)^{a}\right]^{b} h^{G M O K w G}(t) \\
& -(1 / \theta) \bar{\alpha}(a-1) g(t) G(t)^{-1}(t)\left[1-G(t)^{a}\right]^{b} h^{G M O K w G}(t) \\
& +(1 / \theta) \bar{\alpha} a(b-1) g(t) G(t)^{a-1}(t)\left\{1-G(t)^{a}\right\}^{b-1} h^{G M O K w G}(t)+\left(1 / \theta^{2}\right)\left\{\left[1-G(t)^{a}\right]^{b} \bar{\alpha} h^{G M O K w G}(t)\right\}^{2}
\end{aligned}
$$

\subsection{Stochastic orderings}

Here we use the concept of stochastic ordering (Shaked and Shanthikumar, 2007) to derive different ordering conditions on parameters for random variables following GMOKw - G $(\theta, \alpha, a, b)$ distributions.

Let $X$ and $Y$ be two random variables with cfds $\mathrm{F}$ and $\mathrm{G}$, respectively, survival functions $\bar{F}=1-F$ and $\bar{G}=1-G$, and corresponding pdf's $f, g$. Then $X$ is said to be smaller than $Y$ in the likelihood ratio order ( $X \leq_{l r} Y$ ) if $f(t) / g(t)$ is decreasing in $t \geq 0$; stochastic order ( $X \leq_{s t} Y$ ) if $\bar{F}(t) \leq \bar{G}(t)$ for all $t \geq 0$; hazard rate order $\left(X \leq_{h r} Y\right)$ if $\bar{F}(t) / \bar{G}(t)$ is decreasing in $t \geq 0$; reversed hazard rate order $\left(X \leq_{r h r} Y\right.$ ) if $F(t) / G(t)$ is decreasing in $t \geq 0$. These four stochastic orders are related to each other, as $X \leq_{r h r} Y \Leftarrow X \leq_{l r} Y \Rightarrow X \leq_{h r} Y \Rightarrow X \leq_{s t} Y$

\section{Theorem 2:}

Let $X \sim \mathrm{GMOKw}-\mathrm{G}\left(\theta, \alpha_{1}, a, b\right)$ and $Y \sim \mathrm{GMOKw}-\mathrm{G}\left(\theta, \alpha_{2}, a, b\right)$. If $\alpha_{1}<\alpha_{2}$, then $X \leq_{l r} Y$. (See Appendix A.3 for a proof). As a consequence it follows that $X \leq_{h r} Y, X \leq_{r h r} Y$ and $X \leq_{s t} Y$ 


\section{Estimation}

\subsection{Maximum likelihood method}

The maximum likelihood method is the most widely use method of parameter estimation having many desirable properties. This method provides ease of derivation of confidence intervals using asymptotic normality and also likelihood ratio test to discriminate nested models. Here we consider the estimation of the unknown parameters by maximum likelihood.

Let $t=\left(t_{1}, t_{2}, \ldots, t_{n}\right)^{T}$ be a random sample of size $n$ from GMOKw - G $(\theta, \alpha, a, b)$ with parameter vector $\boldsymbol{\eta}=\left(\theta, \alpha, a, b, \boldsymbol{\beta}^{T}\right)^{T}$, where $\boldsymbol{\beta}=\left(\beta_{1}, \beta_{2}, \ldots, \beta_{q}\right)^{T}$ corresponds to the parameter vector of the baseline distribution $\mathrm{G}$. Then the log-likelihood function for $\boldsymbol{\eta}$ is given by

$$
\begin{aligned}
& \ell=\ell(\boldsymbol{\eta}) \quad=n \log \theta+n \theta \log \alpha+n \log (a b)+\sum_{i=0}^{n} \log \left[g\left(t_{i}, \boldsymbol{\beta}\right)\right]+(a-1) \sum_{i=0}^{n} \log \left[G\left(t_{i}, \boldsymbol{\beta}\right)\right] \\
& +(b \theta-1) \sum_{i=0}^{n} \log \left[1-G\left(t_{i}, \boldsymbol{\beta}\right)^{a}\right]-(\theta+1) \sum_{i=1}^{n} \log \left[1-\bar{\alpha}\left[1-G\left(t_{i}, \boldsymbol{\beta}\right)^{a}\right]^{b}\right]
\end{aligned}
$$

The normal equations can be obtained by taking the first derivatives of the log-likelihood function with respect to $\theta, \alpha, a, b$ and $\boldsymbol{\beta}$ and equating them to zeros as follows:

The components of the score vector $U_{\eta}=\left(U_{\theta}, U_{\alpha}, U_{a}, U_{b}, U_{\beta^{T}}\right)^{T}$

$$
\begin{aligned}
& U_{\theta}= \frac{\partial \ell}{\partial \theta}=\frac{n}{\theta}+n \log \alpha+b \sum_{i=0}^{n} \log \left[1-G\left(t_{i}, \beta\right)^{a}\right]-\sum_{i=1}^{n} \log \left[1-\bar{\alpha}\left[1-G\left(t_{i}, \beta\right)^{a}\right]^{b}\right] \\
& U_{\alpha}=\frac{\partial \ell}{\partial \alpha}= \frac{n \theta}{\alpha}-(\theta+1) \sum_{i=0}^{n} \frac{\left[1-G\left(t_{i}, \boldsymbol{\beta}\right)^{a}\right]^{b}}{1-\bar{\alpha}\left[1-G\left(t_{i}, \boldsymbol{\beta}\right)^{a}\right]^{b}} \\
& U_{a}= \frac{\partial \ell}{\partial a}=\frac{n}{a}+\sum_{i=0}^{n} \log \left[G\left(t_{i}, \boldsymbol{\beta}\right)\right]+(1-b \theta) \sum_{i=0}^{n} \frac{G\left(t_{i}, \boldsymbol{\beta}\right)^{a} \log \left[G\left(t_{i}, \boldsymbol{\beta}\right)\right]}{1-G\left(t_{i}, \boldsymbol{\beta}\right)^{a}} \\
&-(\theta+1) \sum_{i=0}^{n} \frac{b \bar{\alpha}\left[1-G\left(t_{i}, \boldsymbol{\beta}\right)^{a}\right]^{b-1} G\left(t_{i}, \boldsymbol{\beta}\right)^{a} \log \left[G\left(t_{i}, \boldsymbol{\beta}\right)\right]}{1-\bar{\alpha}\left[1-G\left(t_{i}, \boldsymbol{\beta}\right)^{a}\right]^{b}} \\
& U_{b}= \frac{\partial \ell}{\partial b}=\frac{n}{b}+\theta \sum_{i=0}^{n} \log \left[1-G\left(t_{i}, \boldsymbol{\beta}\right)^{a}\right]+(\theta+1) \sum_{i=0}^{n} \frac{\bar{\alpha}\left[1-G\left(t_{i}, \boldsymbol{\beta}\right)^{a}\right]^{b} \log \left[1-G\left(t_{i}, \boldsymbol{\beta}\right)^{a}\right]}{1-\bar{\alpha}\left[1-G\left(t_{i}, \boldsymbol{\beta}\right)^{a}\right]^{b}} \\
& U_{\beta}= \frac{\partial \ell}{\partial \boldsymbol{\beta}}=\sum_{i=0}^{n} \frac{g^{(\boldsymbol{\beta})}\left(t_{i}, \boldsymbol{\beta}\right)}{g\left(t_{i}, \boldsymbol{\beta}\right)}+(a-1) \sum_{i=0}^{n} \frac{G^{(\boldsymbol{\beta})}\left(t_{i}, \boldsymbol{\beta}\right)}{G\left(t_{i}, \boldsymbol{\beta}\right)}+(1-b \theta) \sum_{i=0}^{n} \frac{a G\left(t_{i}, \boldsymbol{\beta}\right)^{a-1} G^{(\boldsymbol{\beta})}\left(t_{i}, \boldsymbol{\beta}\right)}{1-G\left(t_{i}, \boldsymbol{\beta}\right)^{a}} \\
&-(\theta+1) \sum_{i=0}^{n} \frac{b \bar{\alpha}\left[1-G\left(t_{i}, \boldsymbol{\beta}\right)^{a}\right]^{b-1} a G\left(t_{i}, \boldsymbol{\beta}\right)^{a-1} G^{(\boldsymbol{\beta})}\left(t_{i}, \boldsymbol{\beta}\right)}{1-\bar{\alpha}\left[1-G\left(t_{i}, \boldsymbol{\beta}\right)^{a}\right]^{b}}
\end{aligned}
$$

Setting these equations to zero $U_{\eta}=\left(U_{\theta}, U_{\alpha}, U_{a}, U_{b}, U_{\beta^{T}}\right)^{T}=0$ and solving them simultaneously yields the maximum likelihood estimate (MLE) $\hat{\boldsymbol{\eta}}=\left(\hat{\theta}, \hat{\alpha}, \hat{a}, \hat{b}, \hat{\boldsymbol{\beta}}^{T}\right)^{T}$ of $\boldsymbol{\eta}=\left(\theta, \alpha, a, b, \boldsymbol{\beta}^{T}\right)^{T}$. 
Because of its complex form the log likelihood is maximized numerically by employing global optimization methods available with software's like R, SAS, Mathematica.

\subsection{Asymptotic standard error and confidence interval for the mles}

The asymptotic variance-covariance matrix of the MLEs of the parameters can obtained by inverting the Fisher information matrix $\mathrm{I}(\boldsymbol{\eta})$ which can be derived using the second partial derivatives of the log-likelihood function with respect to each parameter. The $i j^{\text {th }}$ elements of $I_{n}(\boldsymbol{\eta})$ are given by $\mathrm{I}_{i j}=-E\left[\partial^{2} l(\boldsymbol{\eta}) / \partial \eta_{i} \partial \eta_{j}\right], \quad i, j=1,2, \cdots, 3+q$

While the exact derivation of the above expectations may not be tractable, one can estimate $\mathrm{I}_{n}(\boldsymbol{\eta})$ by the observed Fisher's information matrix $\hat{\mathrm{I}}_{n}(\hat{\boldsymbol{\eta}})=\left(\hat{\mathrm{I}}_{i j}\right)$, where

$$
\hat{\mathrm{I}}_{i j} \approx\left(-\partial^{2} l(\boldsymbol{\eta}) / \partial \eta_{i} \partial \eta_{j}\right)_{\eta=\hat{\eta}}, \quad i, j=1,2, \cdots, 3+q
$$

Under certain regularity conditions on the parameters as $n \rightarrow \infty$ the asymptotic distribution of $\sqrt{n}(\hat{\boldsymbol{\eta}}-\boldsymbol{\eta})$ is $N_{k}\left(0, V_{n}\right)$ where $V_{n}=\left(v_{j j}\right)=\mathrm{I}_{n}^{-1}(\boldsymbol{\eta})$. The result remains valid if $V_{n}$ is replaced by $\hat{V}_{n}=\hat{\mathrm{I}}^{-1}(\hat{\boldsymbol{\eta}})$. An approximate standard error and $(1-\gamma / 2) 100 \%$ confidence interval for the mle of $j^{\text {th }}$ parameter $\eta_{j}$ are respectively given by $\sqrt{\hat{v}_{j j}}$ and $\hat{\eta}_{j} \pm Z_{\gamma / 2} \sqrt{\hat{v}_{j j}}$, where $Z_{\gamma / 2}$ is the $\gamma / 2$ point of standard normal distribution. We provide the MLE method its large sample standard errors, confidence interval in the case of GMOKw $-\mathrm{E}(\theta, \alpha, a, b, \lambda)$ in Appendix A.4.

\subsection{Estimation by method of moments}

Here moments are not in closed form, so we use the PWMs following (Barreto-Souzai et al., 2013) to provide an alternative estimation method. Now

$$
\begin{aligned}
E\left[1-\bar{\alpha}\left[1-G(t)^{a}\right]^{b}\right] & =\int_{-\infty}^{\infty}\left\{\left[1-\bar{\alpha}\left[1-G(t)^{a}\right]^{b}\right]\right\} \frac{\alpha^{\theta} \theta \operatorname{\theta bg}(t) G(t)^{a-1}\left[1-G(t)^{a}\right]^{b \theta-1}}{\left[1-\bar{\alpha}\left[1-G(t)^{a}\right]^{b}\right]^{\theta+1}} d t \\
& =\int_{-\infty}^{\infty}\left\{\alpha^{\theta} \theta \operatorname{\theta abg}(t) G(t)^{a-1}\left[1-G(t)^{a}\right]^{b \theta-1}\right\} /\left[1-\bar{\alpha}\left[1-G(t)^{a}\right]^{b}\right]^{\theta} d t
\end{aligned}
$$

Let, $u=1-\bar{\alpha}\left[1-G(t)^{a}\right]^{b} \quad$ then $\quad d u=\bar{\alpha} \operatorname{abg}(t) G(t)^{a-1}\left[1-G(t)^{a}\right]^{b-1} d t$

$$
\begin{aligned}
& =\left(\theta \alpha^{\theta} / \bar{\alpha}\right) \int_{\alpha}^{1}((1-u) / \bar{\alpha})^{(\theta-1)} / u^{\theta} d u=\theta(\alpha / \bar{\alpha}) \int_{\alpha}^{\theta} u^{-\theta}(1-u)^{\theta-1} d u \\
& =\theta(\alpha / \bar{\alpha})^{\theta} \int_{\alpha}^{1} u^{-1}(1 / u-1)^{\theta-1} d u
\end{aligned}
$$

Suppose $1 / u-1=v \Rightarrow-d u=u^{2} d v$ 


$$
\begin{array}{ll}
=\theta\left(\frac{\alpha}{\bar{\alpha}}\right)^{\theta} \int_{0}^{(1 / \alpha)-1}(1+v)^{-1} v^{\theta-1} d v & =\theta\left(\frac{\alpha}{\bar{\alpha}}\right)^{\theta} \sum_{i \geq 0}^{(1 / \alpha)-1} \int_{0}^{\theta}(-1)^{i} v^{\theta+i-1} d v \\
=\theta\left(\frac{\alpha}{\bar{\alpha}}\right)^{\theta} \sum_{i \geq 0}(-1)^{i}\left[\frac{v^{\theta+i}}{\theta+i}\right]_{0}^{\alpha / \alpha} & =\theta(-\alpha / \bar{\alpha})^{\theta} \sum_{i \geq 0}\left[(-\bar{\alpha} / \alpha)^{\theta+i} /(\theta+i)\right] \\
=(-1)^{\theta} \theta \sum_{i \geq 0}\left[(-\bar{\alpha} / \alpha)^{i} /(\theta+i)\right], \alpha>1 / 2 &
\end{array}
$$

In particular we get the following result for the MOKw-G( $\alpha, a, b)$ (Handique et al., 2017) by putting $\theta=1$.

$$
\begin{aligned}
& E\left[1-\bar{\alpha}\left[1-G(t)^{a}\right]^{b}\right] \\
& =\left(\frac{\alpha}{\bar{\alpha}}\right) \sum_{i \geq 0}\left[\frac{(-\bar{\alpha} / \alpha)^{i+1}}{i+1}\right]=\left(\frac{\alpha}{\bar{\alpha}}\right)\left[\log \left(1+\frac{\bar{\alpha}}{\alpha}\right)\right]=\left(\frac{\alpha}{\bar{\alpha}}\right)\left[-\log \left(\frac{1}{\alpha}\right)\right]=-\left(\frac{\alpha}{1-\alpha}\right) \log (\alpha)
\end{aligned}
$$

Again

$$
\begin{aligned}
& E\left\{\left[1-\bar{\alpha}\left[1-G(t)^{a}\right]^{b}\right]\right\}^{\nu}=\int_{-\infty}^{\infty}\left\{\left[1-\bar{\alpha}\left[1-G(t)^{a}\right]^{b}\right]\right\}^{\nu} \frac{\alpha^{\theta} \theta a b g(t) G(t)^{a-1}\left[1-G(t)^{a}\right]^{b \theta-1}}{\left[1-\bar{\alpha}\left[1-G(t)^{a}\right]^{b}\right]^{\theta+1}} d t \\
& =\int_{-\infty}^{\infty}\left\{\alpha^{\theta} \operatorname{\theta abg}(t) G(t)^{a-1}\left[1-G(t)^{a}\right]^{b \theta-1}\right\} /\left[1-\bar{\alpha}\left[1-G(t)^{a}\right]^{b}\right]^{\theta+1-v} d t
\end{aligned}
$$

Substituting $u=1-\bar{\alpha}\left[1-G(t)^{a}\right]^{b} \quad$ we get $\quad d u=\bar{\alpha} \operatorname{abg}(t) G(t)^{a-1}\left[1-G(t)^{a}\right]^{b-1} d t$

$$
=\frac{\theta \alpha^{\theta}}{\bar{\alpha}} \int_{\alpha}^{1} \frac{((1-u) / \bar{\alpha})^{(\theta-1)}}{u^{\theta+1-v}} d u=\theta\left(\frac{\alpha}{\bar{\alpha}}\right)^{\theta} \int_{\alpha}^{1} u^{v-\theta-1}(1-u)^{\theta-1} d u=\theta\left(\frac{\alpha}{\bar{\alpha}}\right)^{\theta} B_{\alpha}(v-\theta, \theta)
$$

for $\alpha<1, v>\theta \quad$ where $B_{x}(m, n)=\int_{x}^{1} u^{m-1}(1-u)^{n-1} d u$ is the incomplete beta function.

For $\theta=1$ we get the corresponding result of MOKw $-\mathrm{G}(\alpha, a, b)$

$$
E\left\{\left[1-\bar{\alpha}\left[1-G(t)^{a}\right]^{b}\right]\right\}^{v}=\alpha\left(1-\alpha^{\nu-1}\right) / \bar{\alpha}(v-1) .
$$

Therefore we have

$$
E\left[\left[1-\bar{\alpha}\left[1-G(t)^{a}\right]^{b}\right]^{v}\right]=\left\{\begin{array}{l}
(-1)^{\theta} \theta \sum_{i \geq 0}\left[(-\bar{\alpha} / \alpha)^{i} /(\theta+i)\right] \quad \text { for } \theta<1, \alpha>1 / 2, v=1 \\
\theta(\alpha / \bar{\alpha})^{\theta} B_{\alpha}(v-\theta, \theta), \text { for } \alpha<1, v>\theta, v=2,3, \ldots
\end{array}\right.
$$

Given a random sample $t_{1}, t_{2}, \ldots, t_{n}$ from a population with survival function (6), the parameters can then be estimated by solving the following equations

$$
\frac{1}{n} \sum_{i=1}^{n}\left[\left[1-\bar{\alpha}\left[1-G\left(t_{i}\right)^{a}\right]^{b}\right]^{v}\right]=\left\{\begin{array}{l}
(-1)^{\theta} \theta \sum_{i \geq 0}\left[(-\bar{\alpha} / \alpha)^{i} /(\theta+i)\right] \quad \text { for } \theta<1, \alpha>1 / 2, v=1 \\
\theta(\alpha / \bar{\alpha})^{\theta} B_{\alpha}(v-\theta, \theta), \text { for } \alpha<1, v>\theta, v=2,3, \ldots
\end{array}\right.
$$




\subsection{Real life applications}

We consider the following three real life data sets to illustrate the suitability of the proposed GMOKw - G $(\theta, \alpha, a, b)$ distribution distributions considering Weibull and exponential as our G.

Data Set I: The first data set is a subset of data reported Bekker et al. (2000) which corresponds to the survival times (in years) of a group of patients given chemotherapy treatment alone. This data consists of survival times (in years) for 46 patients.

Data Set II: In this application, we work with the survival times (in days) of 72 guinea pigs infected with virulent tubercle bacilli, observed and reported by Bjerkedal (1960).

Data Set III: This data set consists of 100 observations of breaking stress of carbon fibres (in Gba) given by Nichols and Padgett (2006).

Here we have compared the proposed GMOKw $-\mathrm{G}(\theta, \alpha, a, b)$ distribution with it some of its sub models namely the Marshall-Olkin Kumaraswamy-G ( MOKw-G), Kumaraswamy-G $(\mathrm{Kw}-\mathrm{G})$ and Marshall-Olkin-G ( $\mathrm{MO}-\mathrm{G}$ ) and also with beta- $\mathrm{G}(\mathrm{B}-\mathrm{G})$ model considering $\mathrm{G}$ as Weibull and Exponential. The mles of the parameters are estimated through numerical maximization of log likelihood function. We have also provided their standard errors and 95\% confidence intervals using large sample approach (see appendix A.4). The model with lowest AIC (Akaike Information Criterion) is selected as the best in the lot. AIC $=2 k-2 l$, where $k$ is the number of parameters in the statistical model and $l$ is the maximized value of the log-likelihood function under the considered model.

\section{Likelihood Ratio Test for nested models:}

The GMOKw - G $(\theta, \alpha, a, b)$ distribution reduces to MOKw - G $(\alpha, a, b)$ when $\theta=1$, to $\mathrm{Kw}-\mathrm{G}(a, b)$ if $\alpha=\theta=1$, to MO $-\mathrm{G}(\alpha)$ for $a=1, b=1, \theta=1$.

Here we have employed likelihood ratio criterion to test the following null hypothesis:

(i) $H_{0}: \theta=1$, that is the sample is from MOKw - G $(\alpha, a, b)$ $H_{1}: \theta \neq 1$, that is the sample is GMOKw $-\mathrm{G}(\theta, \alpha, a, b)$.

(ii) $H_{0}: \theta=\alpha=1$, that is the sample is from $\mathrm{Kw}-\mathrm{G}(a, b)$ $H_{1}: \theta \neq 1 \alpha \neq 1$, that is the sample is GMOKw - $\mathrm{G}(\theta, \alpha, a, b)$.

(iii) $H_{0}: \theta=1, a=1, b=1$, that is the sample is from MO - $\mathrm{G}(\alpha)$

$$
H_{1}: \theta \neq 1, a \neq 1, b \neq 1 \text {, that is the sample is } \operatorname{GMOKw}-\mathrm{G}(\theta, \alpha, a, b) \text {. }
$$

Writing $\boldsymbol{\eta}=\left(\theta, \alpha, a, b, \boldsymbol{\beta}^{T}\right)^{T}$ the likelihood ratio test statistic is given by $L R=-2 \ln \left(L\left(\hat{\boldsymbol{\eta}}^{*}\right) / L(\hat{\boldsymbol{\eta}})\right)$, where $\hat{\boldsymbol{\eta}}^{*}$ is the restricted ML estimates under the null hypothesis $H_{0}$ and $\hat{\boldsymbol{\eta}}$ is the unrestricted ML estimates under the alternative hypothesis $H_{1}$. Under the null hypothesis $H_{0}$ the LR criterion follows 
Chi-square distribution with degrees of freedom (df) $\left(d f_{\text {alt }}-d f_{\text {null }}\right)$. The null hypothesis is rejected for $p$-value less than 0.05 . Here $\boldsymbol{\beta}^{T}=(\lambda, \delta)$ when our $\mathrm{G}$ is Weibull $(\lambda, \delta)$ and $\boldsymbol{\beta}^{T}=(\lambda)$ when our $\mathrm{G}$ is Exponential $(\lambda)$.

MLEs and standard errors and 95\% confidence intervals (in parentheses) of the parameters for the fitted model along with the AIC values and Likelihood ratio statistic are presented in Table 1, 2, 3, 4, 5 and 6. The distributions belonging to GMOKw $-\mathrm{G}(\theta, \alpha, a, b)$ family generated the lowest values of the AIC in all the examples. Moreover LR test rejects all the sub models in favour of the GMOKw - G $(\theta, \alpha, a, b)$ except in one case for data set III (see table 5) when $\mathrm{MO}-\mathrm{W}(\alpha, \lambda, \delta)$ is chosen ahead of GMOKw - W $(\theta, \alpha, a, b, \lambda, \delta)$.

Plots of the fitted densities and fitted cdf's along with the observed ones presented Figure 3, 4, 5, 6, 7 and 8 also show that the GMOKw $-\mathrm{G}(\theta, \alpha, a, b)$ family closest fit to all the data sets considered here.

Table 1: MLEs, standard errors, confidence intervals (in parentheses) along with AIC, R.E. and LR(P-value) values for the data set I.

\begin{tabular}{|c|c|c|c|c|c|c|c|c|c|}
\hline Models & $\hat{\theta}$ & $\hat{\alpha}$ & $\hat{a}$ & $\hat{b}$ & $\hat{\lambda}$ & $\hat{\delta}$ & AIC & $\mathrm{RE}$ & $\begin{array}{c}\text { LR } \\
\text { (P-value) }\end{array}$ \\
\hline \multirow[t]{2}{*}{$\begin{array}{c}\mathrm{MO}-\mathrm{W} \\
(\alpha, \lambda, \delta)\end{array}$} & --- & $\begin{array}{r}0.449 \\
(0.451)\end{array}$ & --- & --- & $\begin{array}{c}0.418 \\
(0.308)\end{array}$ & $\begin{array}{c}1.229 \\
(0.247)\end{array}$ & 121.74 & 1.62 & $\begin{array}{c}8.10 \\
(0.04)\end{array}$ \\
\hline & & $(0,1.33)$ & & & $(0,1.02)$ & $(0.74,1.71)$ & & & \\
\hline \multirow[t]{2}{*}{$\begin{array}{l}\mathrm{Kw}-\mathrm{W} \\
(a, b, \lambda, \delta)\end{array}$} & --- & --- & $\begin{array}{r}2.160 \\
(1.584)\end{array}$ & $\begin{array}{c}0.208 \\
(0.204)\end{array}$ & $\begin{array}{r}4.521 \\
(3.900)\end{array}$ & $\begin{array}{r}0.836 \\
(0.196)\end{array}$ & 123.44 & 1.06 & $\begin{array}{r}7.80 \\
(0.02)\end{array}$ \\
\hline & & & $(0,5.26)$ & $(0,0.61)$ & $(0,12.16)$ & $(0.45,1.22)$ & & & \\
\hline \multirow[t]{2}{*}{$\begin{array}{l}\text { MOKw-W } \\
(\alpha, a, b, \lambda, \delta)\end{array}$} & & $\begin{array}{r}0.240 \\
(0.367)\end{array}$ & $\begin{array}{c}0.737 \\
(0.938)\end{array}$ & $\begin{array}{l}0.322 \\
(0.504)\end{array}$ & $\begin{array}{c}0.562 \\
(2.102)\end{array}$ & $\begin{array}{l}1.598 \\
(1.658)\end{array}$ & 125.62 & 1.56 & $\begin{array}{c}7.98 \\
(0.004)\end{array}$ \\
\hline & & $(0,0.95)$ & $(0,2.57)$ & $(0,1.31)$ & $(0,4.68)$ & $(0,4.84)$ & & & \\
\hline \multirow[t]{2}{*}{$\begin{array}{l}\text { GMOKw-W } \\
(\theta, \alpha, a, b, \lambda, \delta)\end{array}$} & $\begin{array}{c}0.239 \\
(0.059)\end{array}$ & $\begin{array}{c}0.004 \\
(0.002)\end{array}$ & $\begin{array}{c}0.518 \\
(0.011)\end{array}$ & $\begin{array}{c}0.244 \\
(0.113)\end{array}$ & $\begin{array}{c}0.111 \\
(0.004)\end{array}$ & $\begin{array}{c}4.112 \\
(0.005)\end{array}$ & 119.64 & --- & --- \\
\hline & $(0.12,0.35)$ & $(0.00008,0.007$ & $(0.49,0.53)$ & $(0.02,0.46)$ & $(0.10,0.11)$ & $(4.10,4.12)$ & & & \\
\hline \multirow[t]{2}{*}{$\begin{array}{l}\mathrm{B}-\mathrm{W} \\
(\theta, \alpha, \lambda, \delta)\end{array}$} & $\begin{array}{c}3.418 \\
(6.538)\end{array}$ & $\begin{array}{r}2.024 \\
(2.856)\end{array}$ & --- & --- & $\begin{array}{c}0.467 \\
(0.807)\end{array}$ & $\begin{array}{c}0.695 \\
(0.555)\end{array}$ & 124.14 & 1.62 & --- \\
\hline & $(0,15.87)$ & $(0,7.62)$ & & & $(0,2.04)$ & $(0,1.78)$ & & & \\
\hline
\end{tabular}




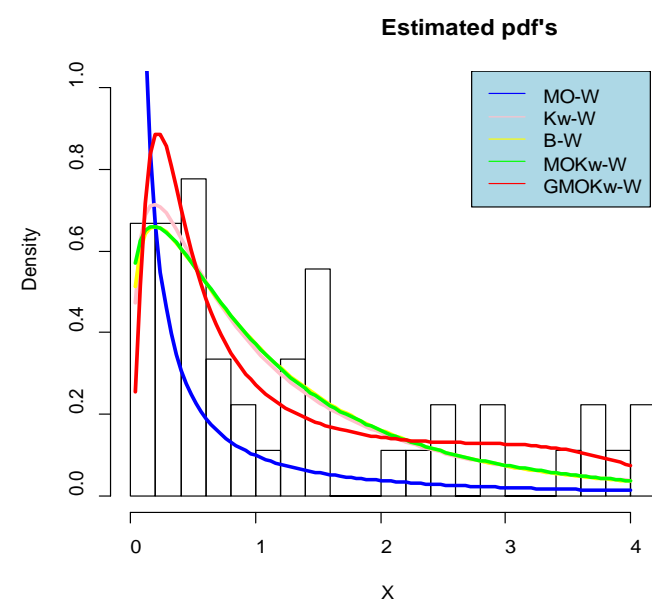

(a)

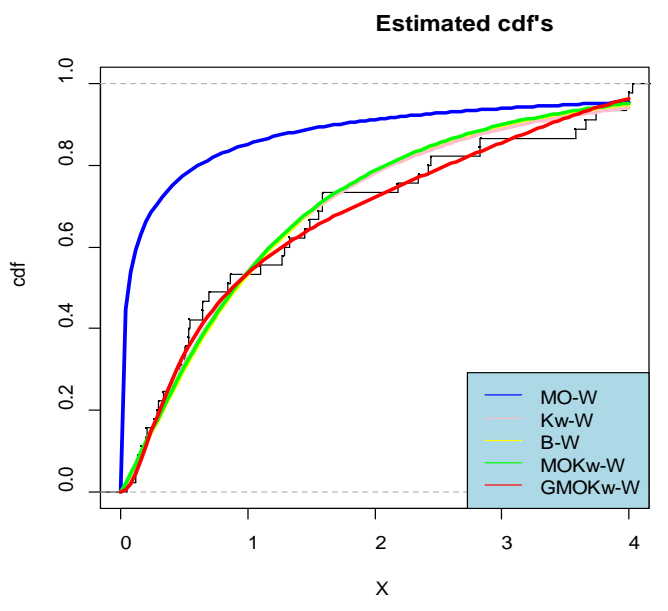

(b)

Fig: 3 Plots of the (a) observed histogram and estimated pdf's and (b) observed ogive and estimated cdf's for the $\mathrm{MO}-\mathrm{W}, \mathrm{Kw}-\mathrm{W}, \mathrm{B}-\mathrm{W}, \mathrm{MOKw}-\mathrm{W}$ and $\mathrm{GMOKw}-\mathrm{W}$ models for the data set $\mathrm{I}$.

Table 2: MLEs, standard errors, confidence intervals (in parentheses) along with AIC, R.E. and LR (P-value) values for the data set I.

\begin{tabular}{|c|c|c|c|c|c|c|c|c|}
\hline Models & $\hat{\theta}$ & $\hat{\alpha}$ & $\hat{a}$ & $\hat{b}$ & $\hat{\lambda}$ & AIC & $\mathrm{RE}$ & $\begin{array}{c}\text { LR } \\
\text { (P-value) }\end{array}$ \\
\hline $\begin{array}{l}\text { MO-E } \\
(\alpha, \lambda)\end{array}$ & --- & $\begin{array}{c}1.022 \\
(0.511) \\
(0.02,2.02)\end{array}$ & --- & --- & $\begin{array}{c}0.752 \\
(0.213) \\
(0.33,1.16)\end{array}$ & 120.54 & 2.11 & $\begin{array}{r}7.92 \\
(0.04)\end{array}$ \\
\hline $\begin{array}{l}\mathrm{Kw}-\mathrm{E} \\
(a, b, \lambda)\end{array}$ & --- & --- & $\begin{array}{c}2.163 \\
(0.539) \\
(1.10,3.21)\end{array}$ & $\begin{array}{c}0.082 \\
(0.012) \\
(0.05,0.11)\end{array}$ & $\begin{array}{c}9.001 \\
(0.002) \\
(8.99,9.004)\end{array}$ & 121.86 & 2.71 & $\begin{array}{c}7.24 \\
(0.03)\end{array}$ \\
\hline $\begin{array}{l}\text { MOKw-E } \\
(\alpha, a, b, \lambda)\end{array}$ & --- & $\begin{array}{c}0.851 \\
(0.501) \\
(0,1.83)\end{array}$ & $\begin{array}{c}2.218 \\
(0.550) \\
(1.14,3.29)\end{array}$ & $\begin{array}{c}0.083 \\
(0.026) \\
(0.03,0.13)\end{array}$ & $\begin{array}{c}8.811 \\
(2.364) \\
(4.17,13.44)\end{array}$ & 122.64 & 1.51 & $\begin{array}{c}6.02 \\
(0.01)\end{array}$ \\
\hline $\begin{array}{l}\text { GMOKw-E } \\
(\theta, \alpha, a, b, \lambda)\end{array}$ & $\begin{array}{c}0.559 \\
(0.494) \\
(0,1.52)\end{array}$ & $\begin{array}{c}0.382 \\
(0.408) \\
(0,1.18)\end{array}$ & $\begin{array}{c}1.427 \\
(0.666) \\
(0.12,2.73)\end{array}$ & $\begin{array}{c}0.852 \\
(0.652) \\
(0,2.12)\end{array}$ & $\begin{array}{c}1.331 \\
(0.992) \\
(0,3.27)\end{array}$ & 118.62 & --- & --- \\
\hline $\begin{array}{l}\mathrm{B}-\mathrm{E} \\
(\theta, \alpha, \lambda)\end{array}$ & $\begin{array}{c}0.119 \\
(0.019) \\
(0.08,0.16)\end{array}$ & $\begin{array}{l}5.110 \\
(8.449) \\
(0,21.67)\end{array}$ & --- & --- & $\begin{array}{c}7.384 \\
(3.814) \\
(0,14.85)\end{array}$ & 120.54 & 1.74 & --- \\
\hline
\end{tabular}




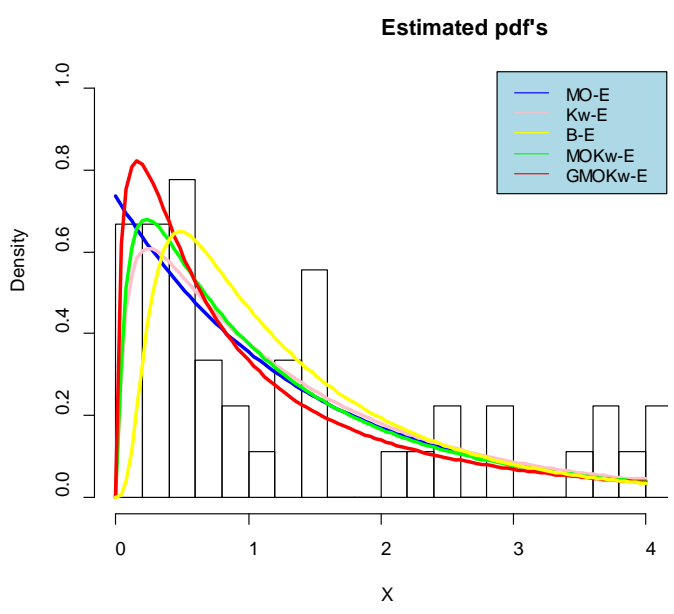

(a)

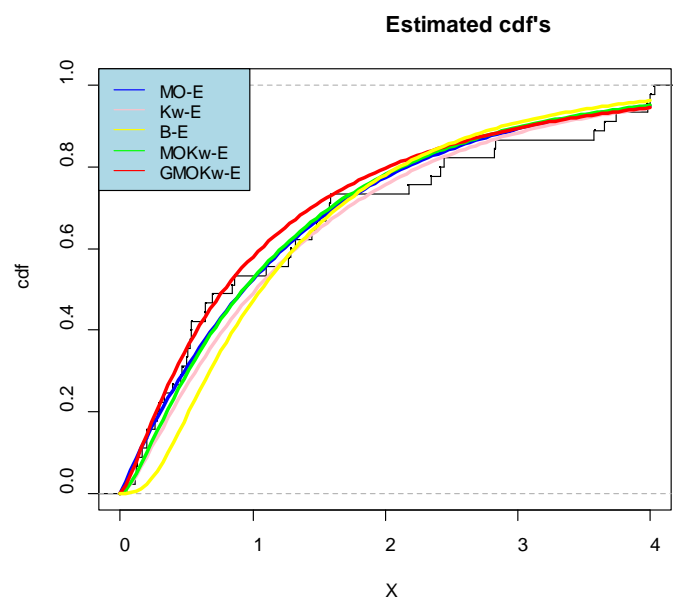

(b)

Fig: 4 Plots of the (a) observed histogram and estimated pdf's and (b) observed ogive and estimated cdf's for the MO - E, Kw - E, B - E , MOKw - E and GMOKw - E models for the data set I.

Table 3: MLEs, standard errors, confidence intervals (in parentheses) along with AIC, R.E. and LR (P-value) values for the data set II.

\begin{tabular}{|c|c|c|c|c|c|c|c|c|c|}
\hline Models & $\hat{\theta}$ & $\hat{\alpha}$ & $\hat{a}$ & $\hat{b}$ & $\hat{\lambda}$ & $\hat{\delta}$ & AIC & $\mathrm{RE}$ & $\begin{array}{c}\text { LR } \\
\text { (P-value) }\end{array}$ \\
\hline $\begin{array}{c}\mathrm{MO}-\mathrm{W} \\
(\alpha, \lambda, \delta)\end{array}$ & --- & $\begin{array}{c}0.032 \\
(0.025) \\
(0,0.08)\end{array}$ & --- & --- & $\begin{array}{c}0.009 \\
(0.007) \\
(0,0.02)\end{array}$ & $\begin{array}{c}2.783 \\
(0.264) \\
(2.26,3.30)\end{array}$ & 203.96 & 1.84 & $\begin{array}{l}7.84 \\
(0.04)\end{array}$ \\
\hline $\begin{array}{l}\mathrm{Kw}-\mathrm{W} \\
(a, b, \lambda, \delta)\end{array}$ & --- & --- & $\begin{array}{c}4.527 \\
(5.481) \\
(0,15.26)\end{array}$ & $\begin{array}{c}1.874 \\
(4.077) \\
(0,9.86)\end{array}$ & $\begin{array}{l}1.025 \\
(0.591) \\
(0,2.18)\end{array}$ & $\begin{array}{c}0.775 \\
(0.700) \\
(0,2.14)\end{array}$ & 207.32 & 3.27 & $\begin{array}{l}9.20 \\
(0.01)\end{array}$ \\
\hline $\begin{array}{l}\text { MOKw-W } \\
(\alpha, a, b, \lambda, \delta)\end{array}$ & --- & $\begin{array}{l}0.111 \\
(0.187) \\
(0,0.48)\end{array}$ & $\begin{array}{c}2.553 \\
(1.829) \\
(0,6.13)\end{array}$ & $\begin{array}{c}0.994 \\
(1.389) \\
(0,3.71)\end{array}$ & $\begin{array}{l}0.299 \\
(0.414) \\
(0,1.11)\end{array}$ & $\begin{array}{l}1.278 \\
(0.703) \\
(0,2.65)\end{array}$ & 207.62 & 1.64 & $\begin{array}{c}7.50 \\
(0.006)\end{array}$ \\
\hline $\begin{array}{l}\text { GMOKw-W } \\
(\theta, \alpha, a, b, \lambda, \delta)\end{array}$ & $\begin{array}{c}1.394 \\
(0.441) \\
(0.52,2.25)\end{array}$ & $\begin{array}{c}0.100 \\
(0.026) \\
(0.04,0.15)\end{array}$ & $\begin{array}{c}1.787 \\
(0.373) \\
(1.05,2.51)\end{array}$ & $\begin{array}{c}0.369 \\
(0.628) \\
(0,1.59)\end{array}$ & $\begin{array}{c}0.247 \\
(0.010) \\
(0.22,0.26)\end{array}$ & $\begin{array}{c}1.665 \\
(1.654) \\
(0,4.91)\end{array}$ & 202.12 & --- & --- \\
\hline $\begin{array}{l}\mathrm{B}-\mathrm{W} \\
(\theta, \alpha, \lambda, \delta)\end{array}$ & $\begin{array}{c}0.712 \\
(1.077) \\
(0,2.82)\end{array}$ & $\begin{array}{c}3.253 \\
(2.227) \\
(0,7.61)\end{array}$ & --- & --- & $\begin{array}{c}1.271 \\
(0.955) \\
(0,3.14) \\
\end{array}$ & $\begin{array}{c}1.041 \\
(0.424) \\
(0.20,1.87) \\
\end{array}$ & 207.38 & 3.28 & --- \\
\hline
\end{tabular}




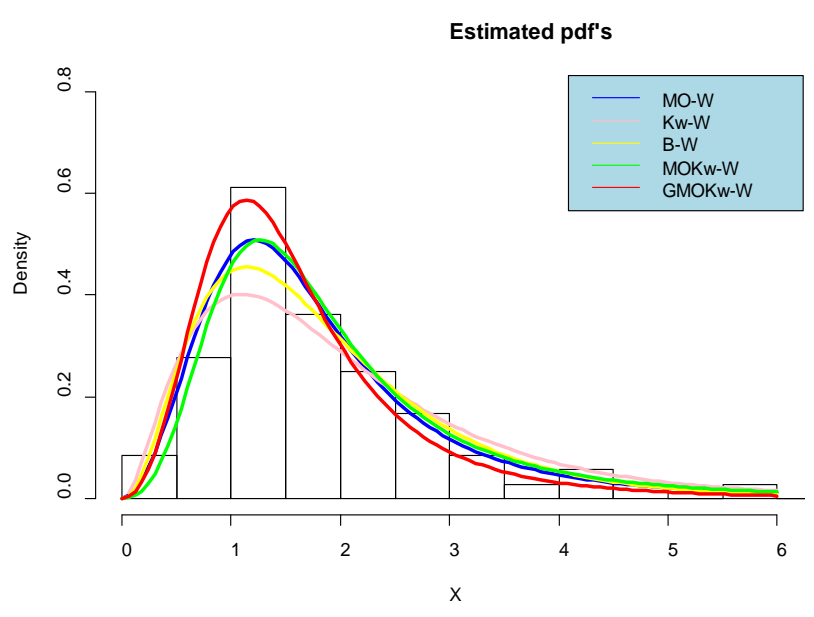

(a)

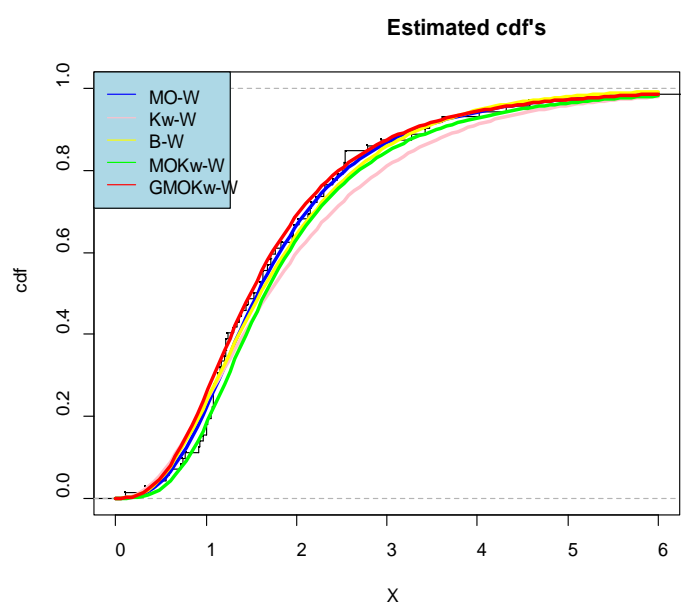

(b)

Fig: 5 Plots of the (a) observed histogram and estimated pdf's and (b) observed ogive and estimated cdf's for the $\mathrm{MO}-\mathrm{W}, \mathrm{Kw}-\mathrm{W}, \mathrm{B}-\mathrm{W}, \mathrm{MOKw}-\mathrm{W}$ and GMOKw $-\mathrm{W}$ models for the data set II.

Table 4: MLEs, standard errors, confidence intervals (in parentheses) along with AIC, R.E. and LR (P-value) values for the data set II.

\begin{tabular}{|c|c|c|c|c|c|c|c|c|}
\hline Models & $\hat{\theta}$ & $\hat{\alpha}$ & $\hat{a}$ & $\hat{b}$ & $\hat{\lambda}$ & AIC & $\mathrm{RE}$ & $\begin{array}{c}\text { LR } \\
\text { (P-value) }\end{array}$ \\
\hline \multirow[t]{2}{*}{$\begin{array}{l}\text { MO-E } \\
(\alpha, \lambda)\end{array}$} & --- & $\begin{array}{c}.778 \\
(3.555)\end{array}$ & --- & --- & $\begin{array}{l}1.379 \\
(0.193)\end{array}$ & 210.36 & 3.58 & $\begin{array}{c}13.12 \\
(0.004)\end{array}$ \\
\hline & & $(1.81,15.74)$ & & & $(1.00,1.75)$ & & & \\
\hline \multirow[t]{2}{*}{$\begin{array}{l}\mathrm{Kw}-\mathrm{E} \\
(a, b, \lambda)\end{array}$} & --- & --- & $\begin{array}{c}3.304 \\
(1.106)\end{array}$ & $\begin{array}{l}1.00 \\
(0.764)\end{array}$ & $\begin{array}{l}1.037 \\
(0.614)\end{array}$ & 209.42 & 2.05 & $\begin{array}{l}10.18 \\
(0.006)\end{array}$ \\
\hline & & & $(1.13,5.47)$ & $(0,2.49)$ & $(0,2.24)$ & & & \\
\hline \multirow[t]{2}{*}{$\begin{array}{l}\mathrm{MOKw}-\mathrm{E} \\
(\alpha, a, b, \lambda)\end{array}$} & --- & $\begin{array}{c}0.008 \\
(0.002)\end{array}$ & $\begin{array}{c}2.716 \\
(1.316)\end{array}$ & $\begin{array}{l}1.986 \\
(0.784)\end{array}$ & $\begin{array}{c}0.086 \\
(0.048)\end{array}$ & 209.44 & 2.47 & $\begin{array}{c}8.20 \\
(0.004)\end{array}$ \\
\hline & & $(0.004,0.01)$ & $(0.14,5.29)$ & $(0.449,3.52)$ & $(0,0.18)$ & & & \\
\hline \multirow[t]{2}{*}{$\begin{array}{l}\text { GMOKw-E } \\
(\theta, \alpha, a, b, \lambda)\end{array}$} & $\begin{array}{c}1.616 \\
(1.086)\end{array}$ & $\begin{array}{c}0.005 \\
(0.003)\end{array}$ & $\begin{array}{c}2.855 \\
(0.504)\end{array}$ & $\begin{array}{l}1.386 \\
(6.534)\end{array}$ & $\begin{array}{l}0.075 \\
(0.151)\end{array}$ & 203.24 & --- & --- \\
\hline & $(0,3.74)$ & $(0,0.01)$ & $(1.86,3.84)$ & $(0,14.19)$ & $(0,0.37)$ & & & \\
\hline \multirow[t]{2}{*}{$\begin{array}{l}\mathrm{B}-\mathrm{E} \\
(\theta, \alpha, \lambda)\end{array}$} & $\begin{array}{l}0.807 \\
(0.696)\end{array}$ & $\begin{array}{c}3.461 \\
(1.003)\end{array}$ & --- & --- & $\begin{array}{c}1.231 \\
(0.855)\end{array}$ & 207.38 & 1.97 & --- \\
\hline & $(0,2.17)$ & $(1.49,5.42)$ & & & $(0,2.91)$ & & & \\
\hline
\end{tabular}




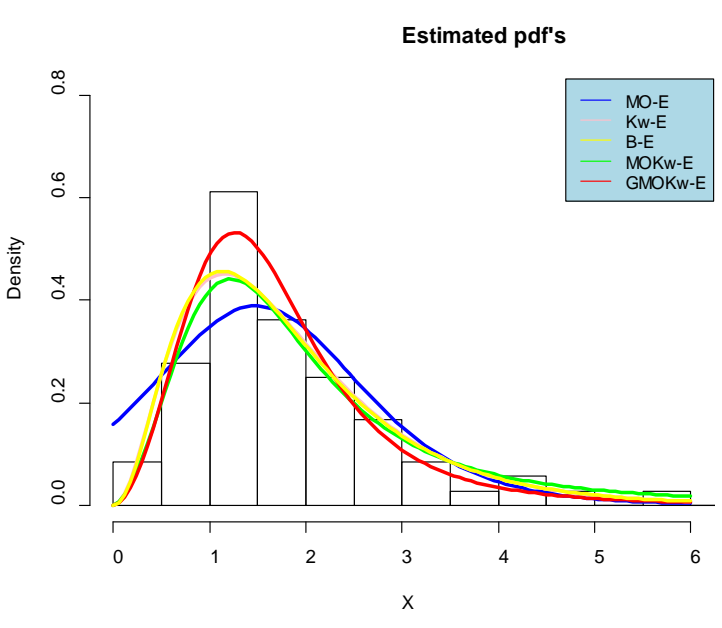

(a)

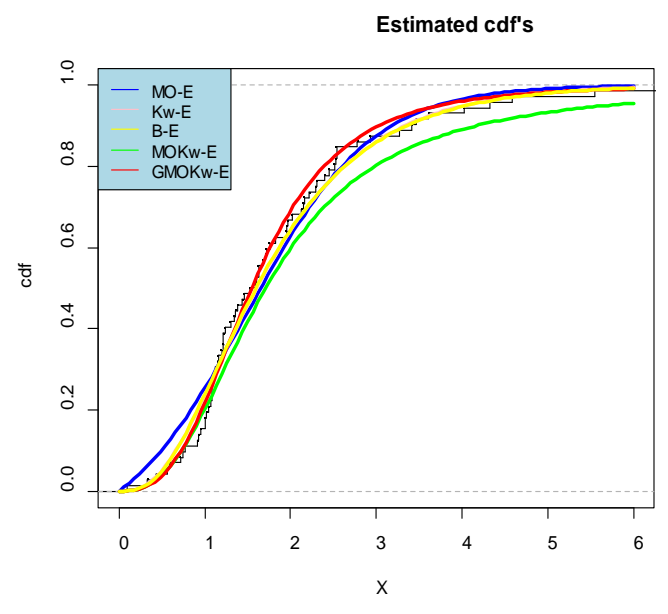

(b)

Fig: 6 Plots of the (a) observed histogram and estimated pdf's and (b) observed ogive and estimated cdf's for the MO - E, Kw - E, B - E , MOKw - E and GMOKw - E models for the data set II.

Table 5: MLEs, standard errors, confidence intervals (in parentheses) along with AIC, R.E. and LR (P-value) values for the data set III.

\begin{tabular}{|c|c|c|c|c|c|c|c|c|c|}
\hline Models & $\hat{\theta}$ & $\hat{\alpha}$ & $\hat{a}$ & $\hat{b}$ & $\hat{\lambda}$ & $\hat{\delta}$ & AIC & $\mathrm{RE}$ & $\begin{array}{c}\text { LR } \\
\text { (P-value) }\end{array}$ \\
\hline \multirow[t]{2}{*}{$\begin{array}{c}\text { MO-W } \\
(\alpha, \lambda, \delta)\end{array}$} & --- & $\begin{array}{c}0.692 \\
(0.677)\end{array}$ & --- & --- & $\begin{array}{c}0.031 \\
(0.039)\end{array}$ & $\begin{array}{c}3.009 \\
(0.601)\end{array}$ & 297.96 & 1.35 & $\begin{array}{c}6.70 \\
(0.08)\end{array}$ \\
\hline & & $(0,2.02)$ & & & $(0,0.10)$ & $(1.83,4.18)$ & & & \\
\hline \multirow[t]{2}{*}{$\begin{array}{l}\mathrm{Kw}-\mathrm{W} \\
(a, b, \lambda, \delta)\end{array}$} & --- & --- & $\begin{array}{c}2.426 \\
(1.297)\end{array}$ & $\begin{array}{r}3.359 \\
(3.054)\end{array}$ & $\begin{array}{c}0.168 \\
(0.148)\end{array}$ & $\begin{array}{l}1.522 \\
(0.573)\end{array}$ & 299.42 & 1.45 & $\begin{array}{r}6.16 \\
(0.04)\end{array}$ \\
\hline & & & $(0,4.96)$ & $(0,9.34)$ & $(0,0.49)$ & $(0.39,2.64)$ & & & \\
\hline \multirow[t]{2}{*}{$\begin{array}{l}\text { MOKw-W } \\
(\alpha, a, b, \lambda, \delta)\end{array}$} & --- & $\begin{array}{c}1.564 \\
(0.550)\end{array}$ & $\begin{array}{r}1.219 \\
(0.108)\end{array}$ & $\begin{array}{c}0.177 \\
(0.031)\end{array}$ & $\begin{array}{c}0.528 \\
(0.002)\end{array}$ & $\begin{array}{c}2.466 \\
(0.003)\end{array}$ & 299.74 & 1.98 & $\begin{array}{c}4.48 \\
(0.03)\end{array}$ \\
\hline & & $(0.48,2.64)$ & $(1.01,1.43)$ & $(0.11,0.24)$ & $(0.52,0.53)$ & $(2.46,2.47)$ & & & \\
\hline \multirow{2}{*}{$\begin{array}{l}\text { GMOKw-W } \\
(\theta, \alpha, a, b, \lambda, \delta)\end{array}$} & $\begin{array}{l}0.345 \\
(0.169)\end{array}$ & $\begin{array}{c}1.482 \\
(0.440)\end{array}$ & $\begin{array}{c}1.015 \\
(0.071)\end{array}$ & $\begin{array}{c}0.385 \\
(0.168)\end{array}$ & $\begin{array}{c}0.803 \\
(0.003)\end{array}$ & $\begin{array}{l}2.222 \\
(0.004)\end{array}$ & 297.26 & --- & --- \\
\hline & $(0.01,0.67)$ & $(0.62,2.35)$ & $(0.87,1.15)$ & $(0.05,0.71)$ & $(0.79,0.81)$ & $(2.21,2.23)$ & & & \\
\hline \multirow[t]{2}{*}{$\begin{array}{l}\mathrm{B}-\mathrm{W} \\
(\theta, \alpha, \lambda, \delta)\end{array}$} & $\begin{array}{l}1.339 \\
(6.288)\end{array}$ & $\begin{array}{c}1.342 \\
(0.757)\end{array}$ & --- & ---- & $\begin{array}{c}0.074 \\
(0.273)\end{array}$ & $\begin{array}{c}2.372 \\
(0.850)\end{array}$ & 298.30 & 1.41 & --- \\
\hline & $(0,13.66)$ & $(0,2.83)$ & & & $(0,0.61)$ & $(0.71,4.04)$ & & & \\
\hline
\end{tabular}




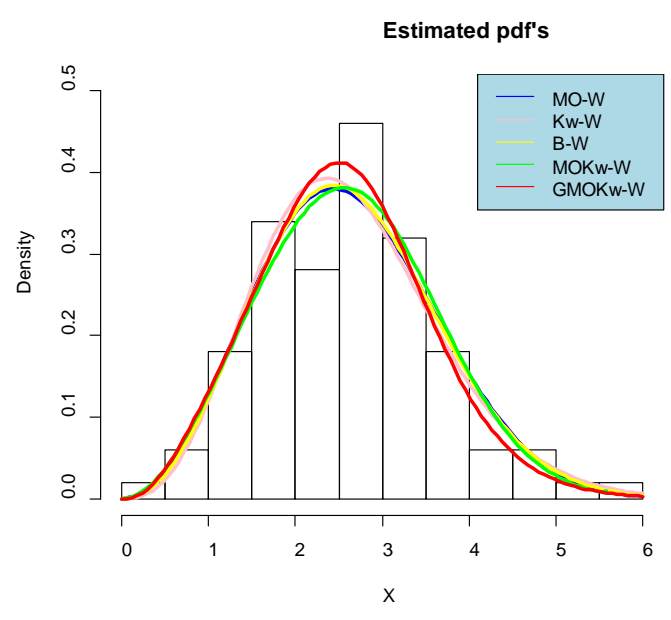

(a)

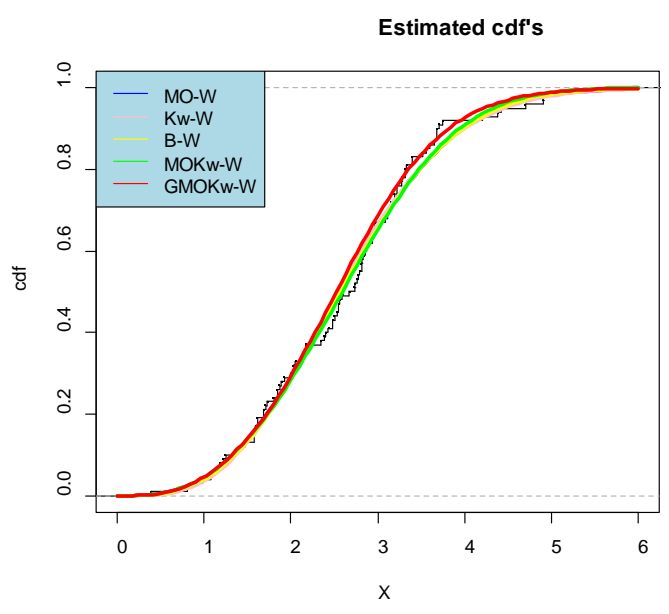

(b)

Fig: 7 Plots of the (a) observed histogram and estimated pdf's and (b) observed ogive and estimated cdf's for the $\mathrm{MO}-\mathrm{W}, \mathrm{Kw}-\mathrm{W}, \mathrm{B}-\mathrm{W}, \mathrm{MOKw}-\mathrm{W}$ and $\mathrm{GMOKw}-\mathrm{W}$ models for the data set III.

Table 6: MLEs, standard errors, confidence intervals (in parentheses) along with AIC, R.E. and LR (P-value) values for the data set III.

\begin{tabular}{|c|c|c|c|c|c|c|c|c|}
\hline Models & $\hat{\theta}$ & $\hat{\alpha}$ & $\hat{a}$ & $\hat{b}$ & $\hat{\lambda}$ & AIC & $\mathrm{RE}$ & $\begin{array}{c}\text { LR } \\
\text { (P-value) }\end{array}$ \\
\hline $\begin{array}{l}\text { MO-E } \\
(\alpha, \lambda)\end{array}$ & --- & $\begin{array}{c}75.663 \\
(33.464) \\
(10.07,141.25)\end{array}$ & --- & --- & $\begin{array}{c}1.535 \\
(0.153) \\
(1.23,1.83)\end{array}$ & 299.04 & 2.51 & $\begin{array}{c}18.78 \\
(0.0003)\end{array}$ \\
\hline $\begin{array}{l}\mathrm{Kw}-\mathrm{E} \\
(a, b, \lambda)\end{array}$ & --- & --- & $\begin{array}{c}3.439 \\
(0.552) \\
(2.35,4.52)\end{array}$ & $\begin{array}{c}48.150 \\
(14.213) \\
(20.29,76.01)\end{array}$ & $\begin{array}{c}0.129 \\
(0.103) \\
(0,0.33)\end{array}$ & 296.64 & 1.23 & $\begin{array}{c}14.38 \\
(0.0007)\end{array}$ \\
\hline $\begin{array}{l}\text { MOKw-E } \\
(\alpha, a, b, \lambda)\end{array}$ & --- & $\begin{array}{c}2.566 \\
(5.709) \\
(0,13.76)\end{array}$ & $\begin{array}{r}3.226 \\
(2.177) \\
(0,7.49)\end{array}$ & $\begin{array}{c}9.065 \\
(42.865) \\
(0,93.08)\end{array}$ & $\begin{array}{c}0.295 \\
(0.834) \\
(0,1.93)\end{array}$ & 290.18 & 1.08 & $\begin{array}{c}5.92 \\
(0.02)\end{array}$ \\
\hline $\begin{array}{l}\text { GMOKw-E } \\
(\theta, \alpha, a, b, \lambda)\end{array}$ & $\begin{array}{c}1.614 \\
(1.215) \\
(0,3.99)\end{array}$ & $\begin{array}{c}6.891 \\
(2.893) \\
(1.22,12.56)\end{array}$ & $\begin{array}{c}3.524 \\
(2.167) \\
(0,7.77)\end{array}$ & $\begin{array}{c}3.363 \\
(12.168) \\
(0,27.21)\end{array}$ & $\begin{array}{c}0.549 \\
(1.217) \\
(0,2.93)\end{array}$ & 286.26 & --- & --- \\
\hline $\begin{array}{c}\mathrm{B}-\mathrm{E} \\
(\theta, \alpha, \lambda)\end{array}$ & $\begin{array}{r}16.575 \\
(13.981) \\
(0,43.97)\end{array}$ & $\begin{array}{c}5.926 \\
(0.825) \\
(4.31,7.54)\end{array}$ & --- & --- & $\begin{array}{c}0.119 \\
(0.090) \\
(0,0.29)\end{array}$ & 292.56 & 1.64 & --- \\
\hline
\end{tabular}




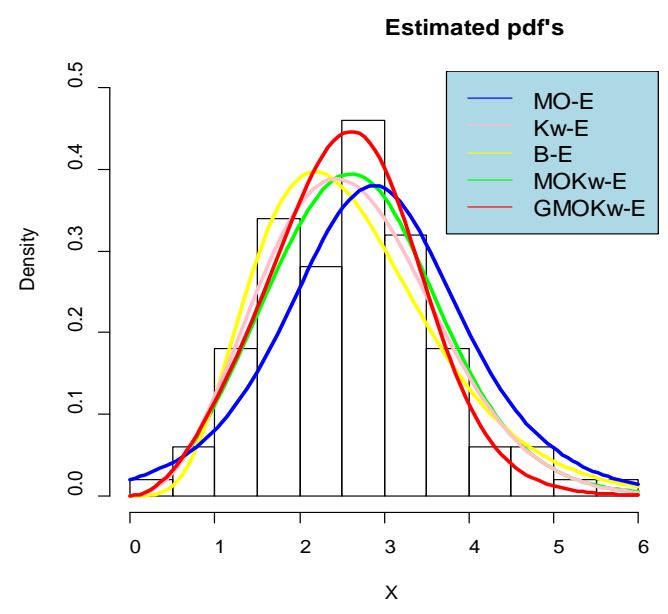

(a)

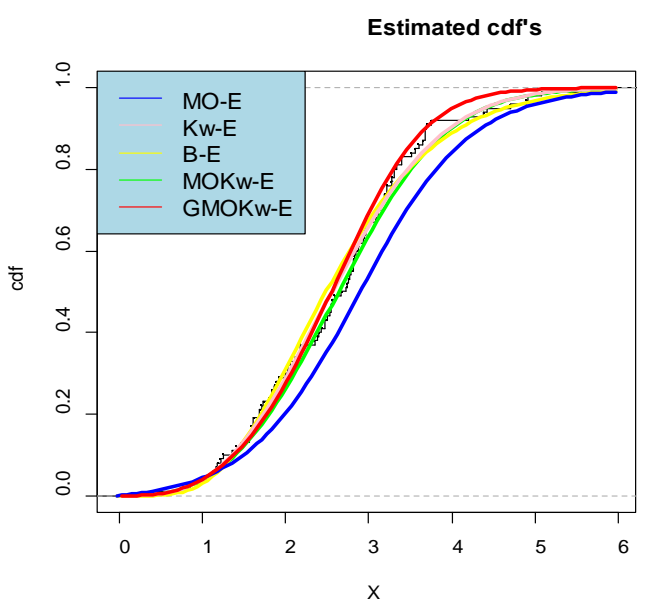

(b)

Fig: 8 Plots of the (a) observed histogram and estimated pdf's and (b) observed ogive and estimated cdf's for the MO - E, Kw - E, B - E , MOKw - E and GMOKw - E models for the data set III.

\section{Conclusion}

A new family of distribution is introduced and its mathematical properties of including order statistics, entropies, and explicit expansions for the order statistics, moments, quantile function, generating function, stochastic ordering, asymptotes, shapes, parameter estimation and data fitting are investigated. Numerical applications with three real life data sets have shown that the distributions from GMOKw - G family provide improved fitting over its sub models.

Acknowledgment Both authors would like to thank the reviewers and the editor for their comments and suggestions.

\section{Appendices}

\section{A.1 Proof of Theorem 1}

Let $\mathrm{T}_{\mathrm{i} 1}, \mathrm{~T}_{\mathrm{i} 2} \ldots, \mathrm{T}_{\mathrm{iN}}$ be a sequence of i.i.d. random variables with survival function $\left[1-G(t)^{a}\right]^{b}$, and suppose $N$ has a geometric distribution with parameter $p$ independent of $T_{i j}$ 's. Then it can easily shown that $W_{i}=\min \left(T_{i 1}, T_{i 2} \ldots, T_{i N}\right) \quad$ and $\quad V_{i}=\max \left(T_{i 1}, T_{i 2} \ldots, T_{i N}\right)$ are distributed as MOKw - G $(p, a, b)$ and MOKw $-\mathrm{G}\left(p^{-1}, a, b\right)$ respectively.

i. For $0<\alpha \leq 1$, if $N$ has a geometric distribution with parameter $\alpha$,then

$$
\begin{aligned}
& P\left[\min \left\{W_{1}, W_{2}, \ldots, W_{\theta}\right\}>t\right]=P\left[W_{1}>t\right] P\left[W_{2}>t\right] \ldots P\left[W_{\theta}>t\right] \\
& =\prod_{i=1}^{\theta} P\left[W_{i}>t\right]=\left[\bar{F}^{\mathrm{MOKwG}}(t ; \alpha, a, b)\right]^{\theta}=\left[\alpha\left[1-G(t)^{a}\right]^{b} / 1-\bar{\alpha}\left[1-G(t)^{a}\right]^{b}\right]^{\theta}
\end{aligned}
$$

This is the sf of GMOKw $-\mathrm{G}(\theta, \alpha, a, b)$.

ii. For $\alpha>1$, if $N$ has a geometric distribution with parameter $\alpha^{-1}$, then 


$$
\begin{aligned}
& P\left[\min \left\{V_{1}, V_{2}, \ldots, V_{\theta}\right\}>t\right]=P\left[V_{1}>t\right] P\left[V_{2}>t\right] \ldots P\left[V_{\theta}>t\right] \\
& =\prod_{i=1}^{\theta} P\left[V_{i}>t\right]=\left[\bar{F}^{\mathrm{MOKwG}}(t ; \alpha, a, b)\right]^{\theta}=\left[\alpha\left[1-G(t)^{a}\right]^{b} / 1-\bar{\alpha}\left[1-G(t)^{a}\right]^{b}\right]^{\theta}\left[\because\left(\alpha^{-1}\right)^{-1}=\alpha\right]
\end{aligned}
$$

\section{A.2 Derivation of pdf and their expansions for order statistics}

Suppose $T_{1}, T_{2}, \ldots T_{n}$ is a random sample from any GMOKw $-\mathrm{G}$ distribution. Let $T_{i: n}$ denote the $i^{\text {th }}$ order statistics. The pdf of $T_{i: n}$ can be expressed as

$$
\begin{aligned}
f_{i: n}(t) & =\{n ! /(i-1) !(n-i) !\} f^{\mathrm{GMOKwG}}(t)\left[1-\bar{F}^{\mathrm{GMOKwG}}(t)\right]^{i-1} \bar{F}^{\mathrm{GMOKwG}}(t)^{n-i} \\
& =\{n ! /(i-1) !(n-i) !\} f^{\mathrm{GMOKwG}}(t) \bar{F}^{\mathrm{GMOKwG}}(t)^{n-i} \sum_{l=0}^{i-1}\{(i-1) ! / l !(i-l-1) !\}\left[-\bar{F}^{\mathrm{GMOKwG}}(t)\right]^{l} \\
& =\{n ! /(i-1) !(n-i) !\} f^{\mathrm{GMOKwG}}(t) \sum_{l=0}^{i-1}(-1)^{l}\{(i-1) ! / l !(i-l-1) !\} \bar{F}^{\mathrm{GMOKwG}}(t)^{n+l-i}
\end{aligned}
$$

Now using the general expansion of the GMOKw - G distribution pdf and sf we get the pdf of the $i^{\text {th }}$ order statistics for of the GMOKw $-\mathrm{G}$ for $\alpha \in(0,1)$ as

$$
\begin{aligned}
& f_{i: n}(t)=\{n ! /(i-1) !(n-i) !\}\left[f^{\mathrm{KwG}}(t ; a, b) \sum_{j=0}^{\infty} A_{j}\left[\bar{F}^{\mathrm{KwG}}(t ; a, b)\right]^{j+\theta-1}\right] \\
& \times \sum_{l=0}^{i-1}(-1)^{l}\left(\begin{array}{c}
i-1 \\
l
\end{array}\right)\left[\sum_{p=0}^{\infty} A_{p}^{\prime}\left[\bar{F}^{\mathrm{KwG}}(t ; a, b)\right]^{p+\theta(n+l-i)}\right] \text { where } A_{j} \text { and } A_{p}^{\prime} \text { are defined earlier. } \\
& =\{n ! /(i-1) !(n-i) !\} \sum_{l=0}^{i-1}\left(\begin{array}{c}
i-1 \\
l
\end{array}\right)(-1)^{l} f^{\mathrm{KwG}}(t ; a, b) \sum_{j=0}^{\infty} \sum_{p=0}^{\infty} A_{j} A_{p}^{\prime}\left[\bar{F}^{\mathrm{KwG}}(t ; a, b)\right]^{j+p+\theta(n+l-i+1)-1} \\
& =f^{\mathrm{KwG}}(t ; a, b) \sum_{j, p=0}^{\infty} M_{j, p}\left[\bar{F}^{\mathrm{KwG}}(t ; a, b)\right]^{j+p+\theta(n+l-i+1)-1} \\
& =-\sum_{j, p=0}^{\infty}\left[M_{j, p} /(j+p+\theta(n+l-i+1))\right] \frac{d}{d t}\left[\bar{F}^{\mathrm{KwG}}(t ; a, b)\right]^{j+p+\theta(n+l-i+1)} \\
& \left.=\sum_{j, p=0}^{\infty} M_{j, p}^{\prime} f^{\mathrm{KwG}}(t ; a, b(j+p+\theta(n+l-i+1)))\right]
\end{aligned}
$$

where $M_{j, p}=n A_{j} A_{p}^{\prime}\left(\begin{array}{c}n-1 \\ i-1\end{array}\right) \sum_{l=0}^{i-1}\left(\begin{array}{c}i-1 \\ l\end{array}\right)(-1)^{l}$ and $M_{j, p}^{\prime}=M_{j, p} /(j+p+\theta(n+l-i+1))$

For $\theta=1$, it reduces to pdf of the $i^{\text {th }}$ order statistics for of the MOKw $-\mathrm{G}(\alpha, a, b)$ given by

$$
f_{i: n}(t)=f^{\mathrm{KwG}}(t ; a, b) \sum_{j, c=0}^{\infty} H_{j, c}\left[\bar{F}^{\mathrm{KwG}}(t ; a, b)\right]^{j+c+n+l-i}
$$

where $H_{j, c}=n \kappa_{j} \eta_{c}\left(\begin{array}{c}n-1 \\ i-1\end{array}\right) \sum_{l=0}^{i-1}\left(\begin{array}{c}i-1 \\ l\end{array}\right)(-1)^{l}$ and $\kappa_{j}=\kappa_{j}(\alpha)=(j+1) \alpha(1-\alpha)^{j} ; \eta_{c}=\alpha(1-\alpha)^{c}$ 
Again using the general expansion of the GMOKw - G distribution pdf and sf we get the pdf of the $i^{\text {th }}$ order statistics for of the GMOKw $-\mathrm{G}$ for $\alpha>1$ as

$$
\begin{aligned}
& f_{i: n}(t)=\{n ! /(i-1) !(n-i) !\}\left\{f^{\mathrm{KwG}}(t ; a, b \theta) \sum_{j=0}^{\infty} C_{j}\left\{F^{\mathrm{KwG}}(t ; a, b)\right\}^{j}\right\} \sum_{l=0}^{i-1}(-1)^{l}\{(i-1) ! / l !(i-l-1) !\} \\
& \times\left\{\left[\bar{F}^{\mathrm{KwG}}(t ; a, b)\right]^{\theta} \sum_{k=0}^{\infty} C_{k}^{\prime}\left[F^{\mathrm{KwG}}(t ; a, b)\right]^{k}\right\}^{n+l-i} \\
&=\{n ! /(i-1) !(n-i) !\}\left\{f^{\mathrm{KwG}}(t ; a, b \theta) \sum_{j=0}^{\infty} C_{j}\left\{F^{\mathrm{KwG}}(t ; a, b)\right\}^{j}\right\} \sum_{l=0}^{i-1}(-1)^{l}\{(i-1) ! / l !(i-l-1) !\} \\
& \times\left[\bar{F}^{\mathrm{KwG}}(t ; a, b)\right]^{\theta(n+l-i)}\left[\sum_{k=0}^{\infty} C_{k}^{\prime}\left[F^{\mathrm{KwG}}(t ; a, b)\right]^{k}\right]^{n+l-i}
\end{aligned}
$$

where $C_{j}$ and $C_{k}^{\prime}$ defined in section 4.1

Now, $\quad\left[\sum_{k=0}^{\infty} C_{k}^{\prime}\left[F^{\mathrm{KwG}}(t ; a, b)\right]^{k}\right]^{n+l-i}=\sum_{k=0}^{\infty} d_{n+l-i, k}\left[F^{\mathrm{KwG}}(t ; a, b)\right]^{k} \quad$ (Nadarajah et. al

2015), where $\quad d_{n+l-i, k}=\left(1 / k C_{0}^{\prime}\right) \sum_{h=1}^{k}[h(n+l-i-1)-k] C_{h}^{\prime} d_{n+l-i, k-h}$

Therefore the density function of the $i^{\text {th }}$ order statistics of GMOKw $-\mathrm{G}$ distribution can be expressed as

$f_{\text {i:n }}(t)$

$$
\begin{gathered}
=\{n ! /(i-1) !(n-i) !\}\left\{f^{\mathrm{KwG}}(t ; a, b \theta) \sum_{j=0}^{\infty} C_{j}\left\{F^{\mathrm{KwG}}(t ; a, b)\right\}^{j}\right\} \sum_{l=0}^{i-1}(-1)^{l}\{(i-1) ! / l !(i-l-1) !\} \\
\times\left[\bar{F}^{\mathrm{KwG}}(t ; a, b)\right]^{\theta(n+l-i)} \sum_{k=0}^{\infty} d_{n+l-i, k}\left[F^{\mathrm{KwG}}(t ; a, b)\right]^{k}
\end{gathered}
$$$$
=f^{\mathrm{KwG}}(t ; a, b)\left[\bar{F}^{\mathrm{KwG}}(t ; a, b)\right]^{\theta(n+l-i+1)-1} \sum_{j, k=0}^{\infty} \phi_{j, k}\left\{F^{\mathrm{KwG}}(t ; a, b)\right\}^{j+k}
$$

where $\phi_{j, k}=n C_{j} d_{n+l-i, k}\{(n-1) ! /(i-1) !(n-i) !\} \sum_{l=0}^{i-1}(-1)^{l}\{(i-1) ! / l !(i-l-1) !\}$

\section{A.3 Proof of Theorem 2}

Let $X \sim \mathrm{GMOKw}-\mathrm{G}\left(\theta, \alpha_{1}, a, b\right)$ and $Y \sim \mathrm{GMOKw}-\mathrm{G}\left(\theta, \alpha_{2}, a, b\right)$. If $\alpha_{1}<\alpha_{2}$, then $X \leq{ }_{l r} Y$

$$
\begin{array}{r}
\frac{f(t)}{g(t)}=\frac{\theta \alpha_{1}^{\theta} \operatorname{abg}(t) G(t)^{a-1}\left[1-G(t)^{a}\right]^{b \theta-1} /\left[1-\bar{\alpha}_{1}\left[1-G(t)^{a}\right]^{b}\right]^{\theta+1}}{\theta \alpha_{2}^{\theta} \operatorname{abg}(t) G(t)^{a-1}\left[1-G(t)^{a}\right]^{b \theta-1} /\left[1-\bar{\alpha}_{2}\left[1-G(t)^{a}\right]^{b}\right]^{\theta+1}} \\
=\left(\alpha_{1} / \alpha_{2}\right)^{\theta}\left[1-\bar{\alpha}_{2}\left[1-G(t)^{a}\right]^{b} /\left\{1-\bar{\alpha}_{1}\left[1-G(t)^{a}\right]^{b}\right\}\right]^{\theta+1}
\end{array}
$$


$d / d t(f(t) / g(t))$

$=(\theta+1)\left(\alpha_{1} / \alpha_{2}\right)^{\theta}\left(\alpha_{1}-\alpha_{2}\right) \frac{\left\{1-\bar{\alpha}_{2}\left[1-G(t)^{a}\right]^{b}\right\}^{\theta} a b\left[1-G(t)^{a}\right]^{b-1} G(t)^{a-1} g(t)}{\left\{1-\bar{\alpha}_{1}\left[1-G(t)^{a}\right]^{b}\right\}^{\theta+2}}$

Now this is always less than 0 since $\alpha_{1}<\alpha_{2}$. Hence, $f(t) / g(t)$ is decreasing in $t$. That is $X \leq_{l r} Y$.

A.4 MLE for GMOKw - E $(\theta, \alpha, a, b, \lambda)$

The pdf of the GMOKw - E distribution is given by

$$
f^{\mathrm{GMOKwE}}(t)=\frac{\theta \alpha^{\theta} a b \lambda e^{-\lambda t}\left[1-e^{-\lambda t}\right]^{a-1}\left[1-\left[1-e^{-\lambda t}\right]^{a}\right]^{b \theta-1}}{\left[1-\bar{\alpha}\left[1-\left[1-e^{-\lambda t}\right]^{a}\right]^{b}\right]^{\theta+1}},
$$

$\theta>0, \lambda>0, \alpha>0, a>0, b>0, t>0$

For a random sample of size $n$ from this distribution, the log-likelihood function for the parameter vector $\boldsymbol{\eta}=(\theta, \alpha, a, b, \lambda)^{T}$ is given by

$$
\begin{aligned}
& \ell=\ell(\boldsymbol{\eta}) \quad=n \log \theta+n \theta \log \alpha+n \log (a b)+n \log \lambda-\lambda \sum_{i=0}^{n} t_{i}+(a-1) \sum_{i=0}^{n} \log \left(1-e^{-\lambda t_{i}}\right) \\
& +(b \theta-1) \sum_{i=0}^{n} \log \left[1-\left(1-e^{-\lambda t_{i}}\right)^{a}\right]-(\theta+1) \sum_{i=0}^{n} \log \left[1-\bar{\alpha}\left[1-\left(1-e^{-\lambda t_{i}}\right)^{a}\right]^{b}\right]
\end{aligned}
$$

The components of the score vector $\boldsymbol{\eta}=(\theta, \alpha, a, b, \lambda)^{T}$ are

$$
\begin{aligned}
& \frac{\partial \ell(\boldsymbol{\eta})}{\partial \theta}=\frac{n}{\theta}+n \log \alpha+b \sum_{i=0}^{n} \log \left[1-\left(1-e^{-\lambda t_{i}}\right)^{a}\right]-\sum_{i=0}^{n} \log \left[1-\bar{\alpha}\left[1-\left(1-e^{-\lambda t_{i}}\right)^{a}\right]^{b}\right] \\
& \frac{\partial \ell(\boldsymbol{\eta})}{\partial \alpha}=\frac{n \theta}{\alpha}-(\theta+1) \sum_{i=0}^{n} \frac{\left[1-\left(1-e^{-\lambda t_{i}}\right)^{a}\right]^{b}}{1-\bar{\alpha}\left[1-\left(1-e^{-\lambda t_{i}}\right)^{a}\right]^{b}} \\
& \frac{\partial \ell(\boldsymbol{\eta})}{\partial a}=\frac{n}{a}+\sum_{i=0}^{n} \log \left(1-e^{-\lambda t_{i}}\right)+(1-b \theta) \sum_{i=0}^{n} \frac{\left(1-e^{-\lambda t_{i}}\right)^{a} \log \left(1-e^{-\lambda t_{i}}\right)}{1-\left(1-e^{-\lambda t_{i}}\right)^{a}} \\
& -(\theta+1) \sum_{i=0}^{n} \frac{\bar{\alpha} b\left[1-\left(1-e^{-\lambda t_{i}}\right)^{a}\right]^{b-1}\left(1-e^{-\lambda t_{i}}\right)^{a} \log \left[\left(1-e^{-\lambda t_{i}}\right)\right]}{1-\bar{\alpha}\left[1-\left(1-e^{-\lambda t_{i}}\right)^{a}\right]^{b}} \\
& \frac{\partial \ell(\boldsymbol{\eta})}{\partial b}=\frac{n}{b}+\theta \sum_{i=0}^{n} \log \left[1-\left(1-e^{-\lambda t_{i}}\right)^{a}\right]+(\theta+1) \sum_{i=0}^{n} \frac{\bar{\alpha}\left[1-\left(1-e^{-\lambda t_{i}}\right)^{a}\right]^{b} \log \left[1-\left(1-e^{-\lambda t_{i}}\right)^{a}\right]}{1-\bar{\alpha}\left[1-\left(1-e^{-\lambda t_{i}}\right)^{a}\right]^{b}} \\
& \frac{\partial \ell(\boldsymbol{\eta})}{\partial \lambda}=\frac{n}{\lambda}-\sum_{i=0}^{n} t_{i}+(a-1) \sum_{i=0}^{n} \frac{\lambda e^{-\lambda t_{i}}}{1-e^{-\lambda t_{i}}}+(1-b \theta) \sum_{i=0}^{n} \frac{a \lambda\left(1-e^{-\lambda t_{i}}\right)^{a-1} e^{-\lambda t_{i}}}{1-\left(1-e^{-\lambda t_{i}}\right)^{a}} \\
& -(\theta+1) \sum_{i=0}^{n} \frac{\bar{\alpha} a b \lambda\left[1-\left(1-e^{-\lambda t_{i}}\right)^{a}\right]^{b-1}\left(1-e^{-\lambda t_{i}}\right)^{a-1} e^{-\lambda t_{i}}}{1-\bar{\alpha}\left[1-\left(1-e^{-\lambda t_{i}}\right)^{a}\right]^{b}}
\end{aligned}
$$

The asymptotic variance covariance matrix for mles of the unknown parameters $\boldsymbol{\eta}=(\theta, \alpha, a, b, \lambda)^{T}$ of GMOKw $-\mathrm{E}(\theta, \alpha, a, b, \lambda)$ distribution is estimated $\operatorname{by} \hat{\mathbf{I}}_{\mathrm{n}}(\hat{\boldsymbol{\eta}})=\left(-\left(\partial^{2} l(\boldsymbol{\eta}) / \partial \eta_{i} \partial \eta_{j}\right)\right)_{\boldsymbol{\eta}=\hat{\boldsymbol{\eta}}}$ 
where the elements of the information matrix can be derived using the following second partial derivatives:

$$
\begin{aligned}
& \frac{\partial^{2} \ell(\boldsymbol{\eta})}{\partial \theta^{2}}=-\frac{n}{\theta^{2}} ; \frac{\partial^{2} \ell(\boldsymbol{\eta})}{\partial \alpha^{2}}=-\frac{n \theta}{\alpha^{2}}-(\theta+1) \sum_{i=0}^{n} \frac{\left[1-\left(1-e^{-\lambda t_{i}}\right)^{a}\right]^{2 b}}{\left\{1-\bar{\alpha}\left[1-\left(1-e^{-\lambda t_{i}}\right)^{a}\right]^{b}\right\}^{2}} ; \\
& \frac{\partial^{2} \ell(\boldsymbol{\eta})}{\partial a^{2}}=\frac{n}{a}+\sum_{i=0}^{n} \log \left(1-e^{-\lambda t_{i}}\right) \\
& +(1-b \theta) \sum_{i=0}^{n} \frac{\left(1-e^{-\lambda t_{i}}\right)^{2 a} \log \left(1-e^{-\lambda t_{i}}\right)^{2}}{\left\{1-\left(1-e^{-\lambda t_{i}}\right)^{a}\right\}^{2}}+(1-b \theta) \sum_{i=0}^{n} \frac{\left(1-e^{-\lambda t_{i}}\right)^{a} \log \left(1-e^{-\lambda t_{i}}\right)^{2}}{1-\left(1-e^{-\lambda t_{i}}\right)^{a}} \\
& +(\theta+1) \sum_{i=0}^{n} \frac{\bar{\alpha}^{2} b^{2}\left[1-\left(1-e^{-\lambda t_{i}}\right)^{a}\right]^{2(b-1)}\left(1-e^{-\lambda t_{i}}\right)^{2 a} \log \left(1-e^{-\lambda t_{i}}\right)^{2}}{\left\{1-\bar{\alpha}\left[1-\left(1-e^{-\lambda t_{i}}\right)^{a}\right]^{b}\right\}^{2}} \\
& +(\theta+1) \sum_{i=0}^{n} \frac{b(b-1) \bar{\alpha}^{2}\left[1-\left(1-e^{-\lambda t_{i}}\right)^{a}\right]^{b-2}\left(1-e^{-\lambda t_{i}}\right)^{2 a} \log \left(1-e^{-\lambda t_{i}}\right)^{2}}{1-\bar{\alpha}\left[1-\left(1-e^{-\lambda t_{i}}\right)^{a}\right]^{b}} \\
& -(\theta+1) \sum_{i=0}^{n} \frac{b \bar{\alpha}\left[1-\left(1-e^{-\lambda t_{i}}\right)^{a}\right]^{b-1}\left(1-e^{-\lambda t_{i}}\right)^{a} \log \left(1-e^{-\lambda t_{i}}\right)^{2}}{1-\bar{\alpha}\left[1-\left(1-e^{-\lambda t_{i}}\right)^{a}\right]^{b}} \\
& \frac{\partial^{2} \ell(\boldsymbol{\eta})}{\partial b^{2}}=-\frac{n}{b^{2}}+(\theta+1) \sum_{i=0}^{n} \frac{\bar{\alpha}^{2}\left[1-\left(1-e^{-\lambda t_{i}}\right)^{a}\right]^{2 b} \log \left[1-\left(1-e^{-\lambda t_{i}}\right)^{a}\right]^{2}}{\left\{1-\bar{\alpha}\left[1-\left(1-e^{-\lambda t_{i}}\right)^{a}\right]^{b}\right\}^{2}} \\
& +(\theta+1) \sum_{i=0}^{n} \frac{\bar{\alpha}\left[1-\left(1-e^{-\lambda t_{i}}\right)^{a}\right]^{b} \log \left[1-\left(1-e^{-\lambda t_{i}}\right)^{a}\right]^{2}}{1-\bar{\alpha}\left[1-\left(1-e^{-\lambda t_{i}}\right)^{a}\right]^{b}} ; \\
& \frac{\partial^{2} \ell(\boldsymbol{\eta})}{\partial \lambda^{2}}=-\frac{n}{\lambda^{2}}+(a-1) \sum_{i=0}^{n}\left(-\frac{e^{-2 \lambda t_{i}} t_{i}^{2}}{\left(1-e^{-\lambda t_{i}}\right)^{2}}-\frac{e^{-\lambda t_{i}} t_{i}^{2}}{1-e^{-\lambda t_{i}}}\right)+(1-b \theta) \sum_{i=0}^{n} \frac{a^{2}\left(1-e^{-\lambda t_{i}}\right)^{2(a-1)} e^{-2 \lambda t_{i}} t_{i}^{2}}{\left\{1-\left(1-e^{-\lambda t_{i}}\right)^{a}\right\}^{2}} \\
& +(1-b \theta) \sum_{i=0}^{n} \frac{a(a-1)\left(1-e^{-\lambda t_{i}}\right)^{a-2} e^{-2 \lambda t_{i}} t_{i}^{2}}{1-\left(1-e^{-\lambda t_{i}}\right)^{a}}-(1-b \theta) \sum_{i=0}^{n} \frac{a\left(1-e^{-\lambda t_{i}}\right)^{a-1} e^{-\lambda t_{i}} t_{i}{ }^{2}}{1-\left(1-e^{-\lambda t_{i}}\right)^{a}} \\
& +(\theta+1) \sum_{i=0}^{n} \frac{\bar{\alpha}^{2} a^{2} b^{2} e^{-2 \lambda t_{i}}\left(1-e^{-\lambda t_{i}}\right)^{2(a-1)}\left[1-\left(1-e^{-\lambda t_{i}}\right)^{a}\right]^{2(b-1)} t_{i}{ }^{2}}{\left\{1-\bar{\alpha}\left[1-\left(1-e^{-\lambda t_{i}}\right)^{a}\right]^{b}\right\}^{2}} \\
& +(\theta+1) \sum_{i=0}^{n} \frac{a^{2} \bar{\alpha} b(b-1) e^{-2 \lambda t_{i}}\left(1-e^{-\lambda t_{i}}\right)^{2(a-1)}\left[1-\left(1-e^{-\lambda t_{i}}\right)^{a}\right]^{b-2} t_{i}{ }^{2}}{1-\bar{\alpha}\left[1-\left(1-e^{-\lambda t_{i}}\right)^{a}\right]^{b}} \\
& -(\theta+1) \sum_{i=0}^{n} \frac{a(a-1) \bar{\alpha} b e^{-2 \lambda t_{i}}\left(1-e^{-\lambda t_{i}}\right)^{a-2}\left[1-\left(1-e^{-\lambda t_{i}}\right)^{a}\right]^{b-1} t_{i}{ }^{2}}{1-\bar{\alpha}\left[1-\left(1-e^{-\lambda t_{i}}\right)^{a}\right]^{b}} \\
& +(\theta+1) \sum_{i=0}^{n} \frac{a b \bar{\alpha} e^{-\lambda t_{i}}\left(1-e^{-\lambda t_{i}}\right)^{a-1}\left[1-\left(1-e^{-\lambda t_{i}}\right)^{a}\right]^{b-1} t_{i}^{2}}{1-\bar{\alpha}\left[1-\left(1-e^{-\lambda t_{i}}\right)^{a}\right]^{b}} ; \\
& \frac{\partial^{2} \ell(\boldsymbol{\eta})}{\partial \theta \partial \alpha}=\frac{n}{\alpha}-\sum_{i=0}^{n} \frac{\left[1-\left(1-e^{-\lambda t_{i}}\right)^{a}\right]^{b}}{1-\bar{\alpha}\left[1-\left(1-e^{-\lambda t_{i}}\right)^{a}\right]^{b}}
\end{aligned}
$$




$$
\begin{aligned}
\frac{\partial^{2} \ell(\boldsymbol{\eta})}{\partial \theta \partial a} & =-b \sum_{i=0}^{n} \frac{\left(1-e^{-\lambda t_{i}}\right)^{a} \log \left(1-e^{-\lambda t_{i}}\right)}{1-\left(1-e^{-\lambda t_{i}}\right)^{a}} \\
& -\sum_{i=0}^{n} \frac{b \bar{\alpha}\left[1-\left(1-e^{-\lambda t_{i}}\right)^{a}\right]^{b-1}\left(1-e^{-\lambda t_{i}}\right)^{a} \log \left(1-e^{-\lambda t_{i}}\right)}{1-\bar{\alpha}\left[1-\left(1-e^{-\lambda t_{i}}\right)^{a}\right]^{b}}
\end{aligned}
$$

$\frac{\partial^{2} \ell(\boldsymbol{\eta})}{\partial \theta \partial b}=\sum_{i=0}^{n} \log \left[1-\left(1-e^{-\lambda t_{i}}\right)^{a}\right]+\sum_{i=0}^{n} \frac{\bar{\alpha}\left[1-\left(1-e^{-\lambda t_{i}}\right)^{a}\right]^{b} \log \left[1-\left(1-e^{-\lambda t_{i}}\right)^{a}\right]}{1-\bar{\alpha}\left[1-\left(1-e^{-\lambda t_{i}}\right)^{a}\right]^{b}} ;$

$\frac{\partial^{2} \ell(\boldsymbol{\eta})}{\partial \theta \partial \lambda}=-b \sum_{i=0}^{n} \frac{a \lambda\left(1-e^{-\lambda t_{i}}\right)^{a-1} e^{-\lambda t_{i}}}{1-\left(1-e^{-\lambda t_{i}}\right)^{a}}-\sum_{i=0}^{n} \frac{\bar{\alpha} a b \lambda\left[1-\left(1-e^{-\lambda t_{i}}\right)^{a}\right]^{b-1}\left(1-e^{-\lambda t_{i}}\right)^{a-1} e^{-\lambda t_{i}}}{1-\bar{\alpha}\left[1-\left(1-e^{-\lambda t_{i}}\right)^{a}\right]^{b}} ;$

$\frac{\partial^{2} \ell(\boldsymbol{\eta})}{\partial \alpha \partial a}=(\theta+1) \sum_{i=0}^{n} \frac{\bar{\alpha} b\left[1-\left(1-e^{-\lambda t_{i}}\right)^{a}\right]^{2 b-1}\left(1-e^{-\lambda t_{i}}\right)^{2 a} \log \left(1-e^{-\lambda t_{i}}\right)}{\left\{1-\bar{\alpha}\left[1-\left(1-e^{-\lambda t_{i}}\right)^{a}\right]^{b}\right\}^{2}}$

$$
(\theta+1) \sum_{i=0}^{n} \frac{b\left[1-\left(1-e^{-\lambda t_{i}}\right)^{a}\right]^{b-1}\left(1-e^{-\lambda t_{i}}\right)^{a} \log \left(1-e^{-\lambda t_{i}}\right)}{1-\bar{\alpha}\left[1-\left(1-e^{-\lambda t_{i}}\right)^{a}\right]^{b}}
$$

$\frac{\partial^{2} \ell(\boldsymbol{\eta})}{\partial \alpha \partial b}=-(\theta+1) \sum_{i=0}^{n} \frac{\bar{\alpha}\left[1-\left(1-e^{-\lambda t_{i}}\right)^{a}\right]^{2 b} \log \left[1-\left(1-e^{-\lambda t_{i}}\right)^{a}\right]}{\left\{1-\bar{\alpha}\left[1-\left(1-e^{-\lambda t_{i}}\right)^{a}\right]^{b}\right\}^{2}}$

$$
-(\theta+1) \sum_{i=0}^{n} \frac{\left[1-\left(1-e^{-\lambda t_{i}}\right)^{a}\right]^{b} \log \left[1-\left(1-e^{-\lambda t_{i}}\right)^{a}\right]}{1-\bar{\alpha}\left[1-\left(1-e^{-\lambda t_{i}}\right)^{a}\right]^{b}}
$$

$\frac{\partial^{2} \ell(\boldsymbol{\eta})}{\partial \alpha \partial \lambda}=(\theta+1) \sum_{i=0}^{n} \frac{\bar{\alpha} a b e^{-\lambda t_{i}}\left(1-e^{-\lambda t_{i}}\right)^{a-1}\left[1-\left(1-e^{-\lambda t_{i}}\right)^{a}\right]^{2 b-1} t_{i}}{\left\{1-\bar{\alpha}\left[1-\left(1-e^{-\lambda t_{i}}\right)^{a}\right]^{b}\right\}^{2}}$

$$
+(\theta+1) \sum_{i=0}^{n} \frac{a \bar{\alpha} b e^{-\lambda t_{i}}\left(1-e^{-\lambda t_{i}}\right)^{a-1}\left[1-\left(1-e^{-\lambda t_{i}}\right)^{a}\right]^{b-1} t_{i}}{1-\bar{\alpha}\left[1-\left(1-e^{-\lambda t_{i}}\right)^{a}\right]^{b}}
$$

$$
\frac{\partial^{2} \ell(\boldsymbol{\eta})}{\partial a \partial b}=-\theta \sum_{i=0}^{n} \frac{\left(1-e^{-\lambda t_{i}}\right)^{a} \log \left(1-e^{-\lambda t_{i}}\right)}{1-\left(1-e^{-\lambda t_{i}}\right)^{a}}
$$

$$
\begin{aligned}
& -(\theta+1) \sum_{i=0}^{n} \frac{\bar{\alpha}\left(1-e^{-\lambda t_{i}}\right)^{a}\left[1-\left(1-e^{-\lambda t_{i}}\right)^{a}\right]^{b-1} \log \left(1-e^{-\lambda t_{i}}\right)}{1-\bar{\alpha}\left[1-\left(1-e^{-\lambda t_{i}}\right)^{a}\right]^{b}} \\
& -(\theta+1) \sum_{i=0}^{n} \frac{b \bar{\alpha}^{2}\left(1-e^{-\lambda t_{i}}\right)^{a}\left[1-\left(1-e^{-\lambda t_{i}}\right)^{a}\right]^{2 b-1} \log \left(1-e^{-\lambda t_{i}}\right) \log \left[1-\left(1-e^{-\lambda t_{i}}\right)^{a}\right]}{\left\{1-\bar{\alpha}\left[1-\left(1-e^{-\lambda t_{i}}\right)^{a}\right]^{b}\right\}^{2}} \\
& -(\theta+1) \sum_{i=0}^{n} \frac{b \bar{\alpha}\left(1-e^{-\lambda t_{i}}\right)^{a}\left[1-\left(1-e^{-\lambda t_{i}}\right)^{a}\right]^{b-1} \log \left(1-e^{-\lambda t_{i}}\right) \log \left[1-\left(1-e^{-\lambda t_{i}}\right)^{a}\right]}{1-\bar{\alpha}\left[1-\left(1-e^{-\lambda t_{i}}\right)^{a}\right]^{b}}
\end{aligned}
$$

$$
\frac{\partial^{2} \ell(\boldsymbol{\eta})}{\partial a \partial \lambda}=\sum_{i=0}^{n} \frac{e^{-\lambda t_{i}} t_{i}}{1-e^{-\lambda t_{i}}}+(1-b \theta) \sum_{i=0}^{n} \frac{a\left(1-e^{-\lambda t_{i}}\right)^{2 a-1} e^{-\lambda t_{i}} t_{i} \log \left(1-e^{-\lambda t_{i}}\right)}{\left\{1-\left(1-e^{-\lambda t_{i}}\right)^{a}\right\}^{2}}
$$

$$
+(1-b \theta) \sum_{i=0}^{n} \frac{\left(1-e^{-\lambda t_{i}}\right)^{a-1} e^{-\lambda t_{i}} t_{i}}{1-\left(1-e^{-\lambda t_{i}}\right)^{a}}+(1-b \theta) \sum_{i=0}^{n} \frac{a\left(1-e^{-\lambda t_{i}}\right)^{a-1} e^{-\lambda t_{i}} t_{i} \log \left(1-e^{-\lambda t_{i}}\right)}{1-\left(1-e^{-\lambda t_{i}}\right)^{a}}
$$




$$
\begin{aligned}
& -(\theta+1) \sum_{i=0}^{n} \frac{b \bar{\alpha} e^{-\lambda t_{i}}\left(1-e^{-\lambda t_{i}}\right)^{a-1}\left[1-\left(1-e^{-\lambda t_{i}}\right)^{a}\right]^{b-1} t_{i}}{1-\bar{\alpha}\left[1-\left(1-e^{-\lambda t_{i}}\right)^{a}\right]^{b}} \\
& +(\theta+1) \sum_{i=0}^{n} \frac{a b^{2} \bar{\alpha}^{2} e^{-\lambda t_{i}}\left(1-e^{-\lambda t_{i}}\right)^{2 a-1}\left[1-\left(1-e^{-\lambda t_{i}}\right)^{a}\right]^{2(b-1)} t_{i} \log \left(1-e^{-\lambda t_{i}}\right)}{\left\{1-\bar{\alpha}\left[1-\left(1-e^{-\lambda t_{i}}\right)^{a}\right]^{b}\right\}^{2}} \\
& +(\theta+1) \sum_{i=0}^{n} \frac{a b(b-1) \bar{\alpha} e^{-\lambda t_{i}}\left(1-e^{-\lambda t_{i}}\right)^{2 a-1}\left[1-\left(1-e^{-\lambda t_{i}}\right)^{a}\right]^{b-2} t_{i} \log \left(1-e^{-\lambda t_{i}}\right)}{1-\bar{\alpha}\left[1-\left(1-e^{-\lambda t_{i}}\right)^{a}\right]^{b}} \\
& -(\theta+1) \sum_{i=0}^{n} \frac{a b \bar{\alpha} e^{-\lambda t_{i}}\left(1-e^{-\lambda t_{i}}\right)^{a-1}\left[1-\left(1-e^{-\lambda t_{i}}\right)^{a}\right]^{b-1} t_{i} \log \left(1-e^{-\lambda t_{i}}\right)}{1-\bar{\alpha}\left[1-\left(1-e^{-\lambda t_{i}}\right)^{a}\right]^{b}} ; \\
\frac{\partial^{2} \ell(\boldsymbol{\eta})}{\partial b \partial \lambda=} & -\sum_{i=0}^{n} \frac{a e^{-\lambda t_{i}}\left(1-e^{-\lambda t_{i}}\right)^{a-1} t_{i}}{1-\left(1-e^{-\lambda t_{i}}\right)^{a}} \\
& -(\theta+1) \sum_{i=0}^{n} \frac{a \bar{\alpha} e^{-\lambda t_{i}}\left(1-e^{-\lambda t_{i}}\right)^{a-1}\left[1-\left(1-e^{-\lambda t_{i}}\right)^{a}\right]^{b-1} t_{i}}{1-\bar{\alpha}\left[1-\left(1-e^{-\lambda t_{i}}\right)^{a}\right]^{b}} \\
& -(\theta+1) \sum_{i=0}^{n} \frac{a b \bar{\alpha}^{2} e^{-\lambda t_{i}}\left(1-e^{-\lambda t_{i}}\right)^{a-1}\left[1-\left(1-e^{-\lambda t_{i}}\right)^{a}\right]^{2 b-1} t_{i} \log \left[1-\left(1-e^{-\lambda t_{i}}\right)^{a}\right]}{\left\{1-\bar{\alpha}\left[1-\left(1-e^{-\lambda t_{i}}\right)^{a}\right]^{b}\right\}^{2}} \\
& -(\theta+1) \sum_{i=0}^{n} \frac{a b \bar{\alpha} e^{-\lambda t_{i}}\left(1-e^{-\lambda t_{i}}\right)^{a-1}\left[1-\left(1-e^{-\lambda t_{i}}\right)^{a}\right]^{b-1} t_{i} \log \left[1-\left(1-e^{-\lambda t_{i}}\right)^{a}\right]}{1-\bar{\alpha}\left[1-\left(1-e^{-\lambda t_{i}}\right)^{a}\right]^{b}}
\end{aligned}
$$

where $\psi^{\prime}($.$) is the derivative of the digamma function.$ 


\section{References}

[1] Abdelfattah, M., Beih, S. El-D. and Shamsan, AL-G. (2016). The Weibull generalized flexible Weibull extension distribution. Journal of Data Science, 14 (3): 453-478.

[2] Afify, A. Z. , Cordeiro, G.M., Yousof H.M., Alzaatreh, A. and Nofal, Z.M. (2016). The Kumaraswamy Transmuted-G family of distributions: Properties and Applications. Journal of Data Science, 14 (2): 245-270.

[3] Alizadeh, M., Tahir, H.M., Cordeiro, G.M., Zubair, M., and Hamedani, G.G. (2015). The Kumaraswamy Marshal-Olkin family of distributions. Journal of the Egyptian Mathematical Society, 23: 546-557.

[4] Barreto-Souza, W., Lemonte, A.J., and Cordeiro, G.M. (2013). General results for Marshall and Olkin's family of distributions. An Acad Bras Cienc, 85: 3-21.

[5] Bekker, A., Roux, J., and Mostert, P. (2000). A generalization of the compound Rayleigh distribution: using a Bayesian methods on cancer survival times, Commun. Stat. Theory Methods, 29: 1419-1433.

[6] Bjerkedal, T. (1960). Acquisition of Resistance in Guinea Pies infected with Different Doses of Virulent Tubercle Bacilli. American Journal of Hygiene, 72: 130-48.

[7] Cordeiro, G.M., and De Castro, M. (2011). A new family of generalized distributions. J Stat Comput Simul, 81: 883-893.

[8] Cordeiro, G.M., Lemonte, A.J., and Ortega, E.E.M. (2014). The Marshall-Olkin family of distributions: Mathematical properties and new models. J Stat Theor Pract, 8: 343-366.

[9] Greenwood, J.A., Landwehr, J.M., Matalas, N.C. and Wallis, J.R. (1979). Probability weighted moments: definition and relation to parameters of several distributions expressible in inverse form. Water Resour Res, 15: 1049-1054.

[10] Handique, L., Chakraborty, S. and Hamedani, G. G. (2017). The Marshall-OlkinKumaraswamy-G family of distributions. Journal of Statistical Theory and Applications (forthcoming, accepted for publication).

[11] Handique, L., and Chakraborty, S. (2016b). Beta generated Kumaraswamy-G and other new families of distributions. arXiv:1603.00634 [math.ST], Under Review.

[12] Jayakumar, K., and Mathew, T. (2008). On a generalization to Marshall-Olkin scheme and its application to Burr type XII distribution. Stat Pap, 49: 421-439.

[13] Kullback, S. (1959). Information Theory and Statistics. John Wiley \& Sons, New York.

[14] Lazhar, B. (2017). Marshall-Olkin extended generalized Gompertz distribution: Journal of Data Science, 15: 239-266

[15] Lehmann, E.L. (1953). The power of rank tests. Ann Math Statist, 24: 23-43. 
[16] Marshall, A., and Olkin, I. (1997). A new method for adding a parameter to a family of distributions with applications to the exponential and Weibull families. Biometrika, 84: 641-652.

[17] Nadarajah, S., Cordeiro, G.M., and Ortega, E.M.M. (2015). The Zografos-Balakrishnan-G family of distributions: mathematical properties and applications, Commun. Stat. Theory Methods, 44:186-215.

[18] Nichols, M.D. and Padgett, W.J.A. (2006). A bootstrap control chart for Weibull percentiles. Quality and Reliability Engineering International, 22: 141-151.

[19] Shahzadi, A., Mohammad, Z.Y. and Azeem, A. (2017). The Modified Burr III G family of distributions. Journal of Data Science, 15 (1): 41-60.

[20] Shaked, M., and Shanthikumar, J.G. (2007). Stochastic Orders, Springer, New York.

[21] Shusen, P., Broderick, O. O., Yuqi, Q. and Daniel, L. (2016). A generalized class of exponentiated modified Weibull distribution with applications. Journal of Data Science, 14 (4): 585-614.

[22] Song, K.S. (2001). Rényi information, loglikelihood and an intrinsic distribution measure. EM J Stat Plan Infer, 93: 51-69.

[23] Tahir, H.M., and Nadarajah, S. (2015). Parameter induction in continuous univariate distributions: Well-established G families. Anais da Academia Brasileira de Ciências (Annals of the Brazilian Academy of Sciences), 87: 539-568.

[24] Thiago, A. N., Bourguignon, M. and Cordeiro, G.M. (2016). The exponentiated generalized extended exponential distribution. Journal of Data Science, 14 (3): 393-414. 